TRANSACTIONS OF THE

AMERICAN MATHEMATICAL SOCIETY

Volume 359, Number 8, August 2007, Pages 4031-4054

S 0002-9947(07)04149-9

Article electronically published on March 20, 2007

\title{
DIFFERENTIABILITY OF SPECTRAL FUNCTIONS FOR SYMMETRIC $\alpha$-STABLE PROCESSES
}

\author{
MASAYOSHI TAKEDA AND KANEHARU TSUCHIDA
}

\begin{abstract}
Let $\mu$ be a signed Radon measure in the Kato class and define a Schrödinger type operator $\mathcal{H}^{\lambda \mu}=\frac{1}{2}(-\Delta)^{\frac{\alpha}{2}}+\lambda \mu$ on $\mathbb{R}^{d}$. We show that its spectral bound $C(\lambda)=-\inf \sigma\left(\mathcal{H}^{\lambda \mu}\right)$ is differentiable if $\alpha<d \leq 2 \alpha$ and $\mu$ is Green-tight.
\end{abstract}

\section{INTRODUCTION}

Let $\left(\mathcal{E}^{(\alpha)}, \mathcal{D}\left(\mathcal{E}^{(\alpha)}\right)\right), 0<\alpha \leq 2$, be the Dirichlet form generated by a symmetric $\alpha$-stable process and $\mu$ a signed Radon measure in the Kato class. Denote by $\mathcal{H}^{\lambda \mu}$ a Schrödinger type operator $\frac{1}{2}(-\Delta)^{\frac{\alpha}{2}}+\lambda \mu, \lambda \in \mathbb{R}^{1}$, and define its spectral function $C(\lambda)$ by

$$
\begin{aligned}
C(\lambda) & =-\inf \left\{\theta: \theta \in \sigma\left(\mathcal{H}^{\lambda \mu}\right)\right\} \\
& =-\inf \left\{\mathcal{E}^{(\alpha)}(u, u)+\lambda \int_{\mathbb{R}^{d}} \tilde{u}^{2} d \mu: u \in \mathcal{D}\left(\mathcal{E}^{(\alpha)}\right), \int_{\mathbb{R}^{d}} u^{2} d x=1\right\},
\end{aligned}
$$

where $\sigma\left(\mathcal{H}^{\lambda \mu}\right)$ is the spectrum of $\mathcal{H}^{\lambda \mu}$ and $\tilde{u}$ is a quasi-continuous version of $u$. When $\alpha=2$, the symmetric $\alpha$-stable process is nothing but a Brownian motion and $\left(\mathcal{E}^{(\alpha)}, \mathcal{D}\left(\mathcal{E}^{(\alpha)}\right)\right)$ is the classical Dirichlet integral. In this paper, we consider the differentiability of the function $C(\lambda)$.

When $\alpha=2$ and the potential $\mu$ is a function in a certain Kato class, Arendt and Batty 3 proved that the spectral function is differentiable at $\lambda=0$ and its derivative equals zero ([3, Corollary 2.10]). Using a large deviation for additive functionals of the Brownian motion, Wu 33 . obtained a necessary and sufficient condition for the spectral function being differentiable at $\lambda=0$. In 30 and 31] we extended Wu's result to measures in the Kato class. Furthermore, we showed that if $d \leq 4$ and the measure $\mu$ belongs to $\mathcal{K}_{d, 2}^{\infty}$, the spectral function is differentiable on $\mathbb{R}^{1}$. Here the class $\mathcal{K}_{d, 2}^{\infty}$ is the set of Green-tight measures introduced in Zhao 35. The notion of Green-tightness was extended by Chen [7 for a large class of Markov processes including the symmetric $\alpha$-stable process. Denote by $\mathcal{K}_{d, \alpha}^{\infty}$ the set of Green-tight measures corresponding to the symmetric $\alpha$-stable process (see Definition 2.1 (III) below). Then a main objective of this paper is to extend the results in [30 and 31] to the symmetric $\alpha$-stable process. In particular, the main theorem is the following.

Received by the editors February 25, 2004 and, in revised form, August 16, 2005

2000 Mathematics Subject Classification. Primary 60J45, 60J40, 35J10.

Key words and phrases. Symmetric stable process, spectral function, criticality, additive functional, Kato measure.

(C)2007 American Mathematical Society Reverts to public domain 28 years from publication 
Theorem 1.1. If $\alpha<d \leq 2 \alpha$ and $\mu=\mu^{+}-\mu^{-} \in \mathcal{K}_{d, \alpha}^{\infty}-\mathcal{K}_{d, \alpha}^{\infty}$, then the spectral function $C(\lambda)$ is differentiable for all $\lambda \in \mathbb{R}^{1}$.

To prove the differentiability of the spectral function at $\lambda=0$, one of authors used in 30 a well-known property of the Brownian motion; if $d \leq 2$, the Brownian motion is a Harris recurrent process with infinite invariant measure, the Lebesgue measure. However, since the symmetric $\alpha$-stable process is transient for $\alpha<d$, the arguments in [30] cannot be used immediately for the proof of Theorem 1.1. Thus to overcome this, we prepare criticality theory for the Schrödinger type operator $\mathcal{H}^{\lambda \mu}$. More precisely, let

$$
\begin{aligned}
& \lambda^{+}=\inf \{\lambda>0: C(\lambda)>0\}, \\
& \lambda^{-}=\sup \{\lambda<0: C(\lambda)>0\} .
\end{aligned}
$$

We prove that if $\alpha<d$, then the operator $\mathcal{H}^{\lambda^{ \pm} \mu}$ is critical, that is, $\mathcal{H}^{\lambda^{ \pm}} \mu$ does not admit the minimal positive Green function (i.e. non-subcriticality) but admits a positive continuous $\mathcal{H}^{\lambda^{ \pm}} \mu$-harmonic function (this function is called a ground state and uniquely determined up to constant multiplication.). Moreover, we prove that if $d \leq 2 \alpha$, then $\mathcal{H}^{\lambda^{ \pm} \mu}$ is null critical, that is, the ground state does not belong to $L^{2}$. In fact, denoting by $h$ the ground state, we show in section 5 that there exist positive constants $c, C$ such that

$$
\frac{c}{|x|^{d-\alpha}} \leq h(x) \leq \frac{C}{|x|^{d-\alpha}}, \quad|x|>1 .
$$

Using these facts, we find that if $\alpha<d \leq 2 \alpha$, the $h$-transformed process generated by the Markov semigroup

$$
P_{t}^{\lambda^{ \pm} \mu, h} f(x)=\frac{1}{h(x)} \exp \left(-t \mathcal{H}^{\lambda^{ \pm} \mu}\right)(h f)(x),
$$

is a Harris recurrent Markov process with infinite invariant measure $h^{2} d x$. Consequently, the arguments in [30] still work for $\alpha<d \leq 2 \alpha$. This is a key idea of the proof of Theorem 1.1 .

The criticality of Schrödinger type operators has been studied by many people (M. Murata, Y. Pinchover, R. Pinsky,...). The equation (1.1) was shown by Murata [16] for Schrödinger operators on $\mathbb{R}^{d}$ and extended by Pinchover [18] to second order elliptic operators in a domain of $\mathbb{R}^{d}$. If $\mu=0$, criticality and null criticality are equivalent to recurrence and null recurrence respectively.

The equation (1.1) says that if $d>2 \alpha$, then $\mathcal{H}^{\lambda^{ \pm}} \mu$ is positive critical, that is, the ground state belongs to $L^{2}$. Hence the transformed process has a finite invariant measure $h^{2} d x$. Using the argument in 23], we can show that $C(\lambda)$ is not differentiable at $\lambda=\lambda^{ \pm}(6.9)$.

Our motivation lies in the proof of a large deviation principle for the continuous additive functional $A_{t}^{\mu}$ in the Revuz correspondence with $\mu$. The function $C(\lambda)$ is regarded as a logarithmic moment generating function of the additive functional $A^{\mu}$ (see 26]), and the differentiability of logarithmic moment generating functions plays a crucial role in the Gärtner-Ellis Theorem (see [11). When $\alpha=2$ (the Brownian case), $C(\lambda)$ is indeed the logarithmic moment generating function (see 26]). Thus using Theorem 1.1, we can prove the large deviation principle for additive functional $A_{t}^{\mu}$ associated with $\mu=\mu^{+}-\mu^{-} \in \mathcal{K}_{d, 2}^{\infty}-\mathcal{K}_{d, 2}^{\infty}$. However, for general $\alpha$, we have 
not yet known whether $C(\lambda)$ is regarded as the logarithmic moment generating function.

The structure of this paper is the following. In Section 2, we collect necessary preliminary material on measures in the Kato class, and give another characterization of $\lambda^{ \pm}$(Lemma 2.4). This shows the non-subcriticality of $\mathcal{H}^{\lambda^{ \pm}} \mu$. In Section 3 , we prove that the extended Dirichlet space of the symmetric $\alpha$-stable process is compactly embedded in the $L^{2}$-space with respect to a positive Green-tight measure (Theorem 3.4).

In section 4 , we prove the criticality of $\mathcal{H}^{\lambda^{ \pm}} \mu$. To do this, we construct a ground state of $\mathcal{H}^{\lambda^{ \pm}} \mu$ (Lemma 4.9, Lemma 4.10, and Proposition 4.12). If $\alpha=2$, that is, the Shrödinger operator $\mathcal{H}^{\lambda^{ \pm}} \mu$ is a local one, a "standard compactness argument" is applicable for the proof of existence of the ground state (see the Remark below [19, Theorem 3.2]). On the other hand, we do not know whether the "standard compactness argument" is generalized for our non-local operators. Hence we show it by a different method; instead of constructing the ground state of $\mathcal{H}^{\lambda^{ \pm}} \mu$, we first prove the existence of the principal eigenfunction of a time changed process by using the compact embeddness established in Section 3, and then identifying the principal eigenfunction with the ground state. In the proof of the identification, we introduce a new notion of extended Schrödinger spaces, which is regarded as a generalization of the notion of extended Dirichlet spaces. Moreover, we prove that $\mathcal{H}^{\lambda^{ \pm} \mu}$ is null critical if and only if $d \leq 2 \alpha$ (see (4.19)).

In the proof of Theorem 1.1, a functional inequality due to Oshima 17, plays an important role. Oshima's inequality has been derived for a general Dirichlet form generated by symmetric Harris recurrent Markov processes (this is the reason why we need Harris's recurrence). In Section 5, we extend Oshima's inequality to critical Schrödinger forms through $h$-transform (Theorem 5.3). In Section 6, we prove the main theorem.

\section{Preliminaries}

Let $\mathbf{M}^{\alpha}=\left(\Omega, \mathcal{F}, \mathcal{F}_{t}, \theta_{t}, P_{x}, X_{t}\right)$ be a symmetric $\alpha$-stable process on $\mathbb{R}^{d}$ with $0<\alpha \leq 2$. Here $\left\{\mathcal{F}_{t}\right\}_{t \geq 0}$ is the minimal (augmented) admissible filtration and $\theta_{t}$, $t \geq 0$, is the shift operator satisfying $X_{s}\left(\theta_{t}\right)=X_{s+t}$ identically for $s, t \geq 0$. When $\alpha=2, \mathbf{M}^{\alpha}$ is a Brownian motion. We assume that $\alpha<d$, that is, the process $\mathbf{M}^{\alpha}$ is transient. Let $p(t, x, y)$ be the transition density function of $\mathbf{M}^{\alpha}$ and $G(x, y)$ be its Green function:

$$
G(x, y)=\int_{0}^{\infty} p(t, x, y) d t=C(d, \alpha)|x-y|^{\alpha-d},
$$

where $C(d, \alpha)=2^{1-\alpha} \pi^{-d / 2} \Gamma\left(\frac{d-\alpha}{2}\right) \Gamma\left(\frac{\alpha}{2}\right)^{-1}$. For a measure $\mu$, the 0 -potential of $\mu$ is defined by

$$
G \mu(x)=\int_{\mathbb{R}^{d}} G(x, y) \mu(d y) .
$$

Let $P_{t}$ be the semigroup of $\mathbf{M}^{\alpha}$,

$$
P_{t} f(x)=\int_{\mathbb{R}^{d}} p(t, x, y) f(y) d y=E_{x}\left[f\left(X_{t}\right)\right] .
$$


Let $\left(\mathcal{E}^{(\alpha)}, \mathcal{D}\left(\mathcal{E}^{(\alpha)}\right)\right)$ be the Dirichlet form generated by $\mathbf{M}^{\alpha}$. For $0<\alpha<2$, it is given by

$$
\begin{aligned}
\mathcal{E}^{(\alpha)}(u, v) & =\frac{1}{2} \mathcal{A}(d, \alpha) \iint_{\mathbb{R}^{d} \times \mathbb{R}^{d} \backslash d} \frac{(u(x)-u(y))(v(x)-v(y))}{|x-y|^{d+\alpha}} d x d y, \\
\mathcal{D}\left(\mathcal{E}^{(\alpha)}\right) & =\left\{u \in L^{2}\left(\mathbb{R}^{d}\right): \iint_{\mathbb{R}^{d} \times \mathbb{R}^{d} \backslash d} \frac{(u(x)-u(y))^{2}}{|x-y|^{d+\alpha}} d x d y<\infty\right\},
\end{aligned}
$$

where

$$
\mathcal{A}(d, \alpha)=\frac{\alpha 2^{d-1} \Gamma\left(\frac{\alpha+d}{2}\right)}{\pi^{d / 2} \Gamma\left(1-\frac{\alpha}{2}\right)}
$$

([12, Example 1.4.1]). For $\alpha=2$

$$
\begin{aligned}
\mathcal{E}^{(2)}(u, v) & =\frac{1}{2} \mathbf{D}(u, v), \\
\mathcal{D}\left(\mathcal{E}^{(2)}\right) & =H^{1}\left(\mathbb{R}^{d}\right),
\end{aligned}
$$

where $\mathbf{D}$ denotes the classical Dirichlet integral and $H^{1}\left(\mathbb{R}^{d}\right)$ is the Sobolev space of order 1 ([12, Example 4.4.1]). Let $\mathcal{D}_{e}\left(\mathcal{E}^{(\alpha)}\right)$ denote the extended Dirichlet space, that is, the family of measurable functions $u$ on $\mathbb{R}^{d}$ such that $|u|<\infty m$-a.e. and there exists an $\mathcal{E}^{(\alpha)}$-Cauchy sequence $\left\{u_{n}\right\}$ of functions in $\mathcal{D}\left(\mathcal{E}^{(\alpha)}\right)$ such that $\lim _{n \rightarrow \infty} u_{n}=u m$-a.e. $\left(\left[12\right.\right.$, p.35]). Then $\mathcal{D}_{e}\left(\mathcal{E}^{(\alpha)}\right)$ is a Hilbert space with inner product $\mathcal{E}^{(\alpha)}$ because $\mathbf{M}^{\alpha}$ is transient ([12, Theorem 1.5.3]).

Throughout this paper, $m$ is the Lebesgue measure and $B(R)$ is an open ball with radius $R$ centered at the origin. We use $c, C, \ldots$ as positive constants which may be different at different occurrences.

We now define classes of measures which play an important role in this paper.

Definition 2.1. (I) A positive Radon measure $\mu$ on $\mathbb{R}^{d}$ is said to be in the Kato class $\left(\mu \in \mathcal{K}_{d, \alpha}\right.$ in notation), if

$$
\lim _{a \rightarrow 0} \sup _{x \in \mathbb{R}^{d}} \int_{|x-y| \leq a} G(x, y) \mu(d y)=0 .
$$

(II) A measure $\mu$ is in $\mathcal{K}_{d, \alpha}^{\infty}$ if $\mu$ is in $\mathcal{K}_{d, \alpha}$ and satisfies

$$
\lim _{R \rightarrow \infty} \sup _{x \in \mathbb{R}^{d}} \int_{|y|>R} G(x, y) \mu(d y)=0
$$

(cf. [35, Definition 1]).

For $\mu=\mu^{+}-\mu^{-} \in \mathcal{K}_{d, \alpha}-\mathcal{K}_{d, \alpha}$, define a symmetric bilinear form $\mathcal{E}^{\mu}$ by

$$
\mathcal{E}^{\mu}(u, u)=\mathcal{E}^{(\alpha)}(u, u)+\int_{\mathbb{R}^{d}} \widetilde{u}^{2} d \mu, \quad u \in \mathcal{D}\left(\mathcal{E}^{(\alpha)}\right),
$$

where $\widetilde{u}$ is a quasi-continuous version of $u([12$, Theorem 2.1.3]). In the sequel, we always assume that every function $u \in \mathcal{D}_{e}\left(\mathcal{E}^{(\alpha)}\right)$ is represented by its quasi-continuous version. Since $\mu \in \mathcal{K}_{d, \alpha}$ charges no set of zero capacity by [2, Theorem 3.3], the form $\mathcal{E}^{\mu}$ is well defined. We see from [2, Theorem 4.1] that $\left(\mathcal{E}^{\mu}, \mathcal{D}\left(\mathcal{E}^{(\alpha)}\right)\right)$ becomes a lower semi-bounded closed symmetric form. We call $\left(\mathcal{E}^{\mu}, \mathcal{D}\left(\mathcal{E}^{(\alpha)}\right)\right)$ a Schrödinger form. Denote by $\mathcal{H}^{\mu}$ the self-adjoint operator generated by $\left(\mathcal{E}^{\mu}, \mathcal{D}\left(\mathcal{E}^{(\alpha)}\right)\right): \mathcal{E}^{\mu}(u, v)=$ $\left(\mathcal{H}^{\mu} u, v\right)$. Let $P_{t}^{\mu}$ be the $L^{2}$-semigroup generated by $\mathcal{H}^{\mu}: P_{t}^{\mu}=\exp \left(-t \mathcal{H}^{\mu}\right)$. We 
see from [2, Theorem 6.3(iv)] that $P_{t}^{\mu}$ admits a symmetric integral kernel $p^{\mu}(t, x, y)$ which is a jointly continuous function on $(0, \infty) \times \mathbb{R}^{d} \times \mathbb{R}^{d}$.

For $\mu \in \mathcal{K}_{d, \alpha}$, let $A_{t}^{\mu}$ be a positive continuous additive functional which is in the Revuz correspondence with $\mu$ : for any $f \in \mathcal{B}^{+}$and $\gamma$-excessive function $h$,

$$
\langle h \mu, f\rangle=\lim _{t \rightarrow 0} \frac{1}{t} E_{h m}\left(\int_{0}^{t} f\left(X_{s}\right) d A_{s}^{\mu}\right)
$$

([12, p.188]). For $\mu=\mu^{+}-\mu^{-} \in \mathcal{K}_{d, \alpha}-\mathcal{K}_{d, \alpha}$, set $A_{t}^{\mu}=A_{t}^{\mu^{+}}-A_{t}^{\mu^{-}}$. By the Feynman-Kac formula, the semigroup $P_{t}^{\mu}$ is written as

$$
P_{t}^{\mu} f(x)=E_{x}\left[\exp \left(-A_{t}^{\mu}\right) f\left(X_{t}\right)\right] .
$$

The spectral function $C(\lambda)$ is defined by the bottom of the spectrum of $\mathcal{H}^{\lambda \mu}$ : for $\mu=\mu^{+}-\mu^{-} \in \mathcal{K}_{d, \alpha}^{\infty}-\mathcal{K}_{d, \alpha}^{\infty}$,

$$
C(\lambda)=-\inf \left\{\mathcal{E}^{\lambda \mu}(u, u) ; u \in \mathcal{D}\left(\mathcal{E}^{(\alpha)}\right), \int_{\mathbb{R}^{d}} u^{2} d x=1\right\} .
$$

Lemma 2.2. The following statements are equivalent:

(i) $\quad \inf \left\{\mathcal{E}^{(\alpha)}(u, u)+\int_{\mathbb{R}^{d}} u^{2} d \mu^{+}: \int_{\mathbb{R}^{d}} u^{2} d \mu^{-}=1\right\}<1$.

(ii) $\inf \left\{\mathcal{E}^{(\alpha)}(u, u)+\int_{\mathbb{R}^{d}} u^{2} d \mu: \int_{\mathbb{R}^{d}} u^{2} d x=1\right\}<0$.

Proof. Assume (i). Then there exists a $\varphi_{0} \in C_{0}^{\infty}\left(\mathbb{R}^{d}\right)$ with $\int_{\mathbb{R}^{d}} \varphi_{0}^{2} d \mu^{-}=1$ such that

$$
\mathcal{E}^{(\alpha)}\left(\varphi_{0}, \varphi_{0}\right)+\int_{\mathbb{R}^{d}} \varphi_{0}^{2} d \mu^{+}<1
$$

Hence we see that

$$
\mathcal{E}^{(\alpha)}\left(\varphi_{0}, \varphi_{0}\right)+\int_{\mathbb{R}^{d}} \varphi_{0}^{2} d \mu^{+}<\int_{\mathbb{R}^{d}} \varphi_{0}^{2} d \mu^{-} .
$$

Letting

$$
u_{0}=\frac{\varphi_{0}}{\sqrt{\int_{\mathbb{R}^{d}} \varphi_{0}^{2} d x}}
$$

we have

$$
\mathcal{E}^{(\alpha)}\left(u_{0}, u_{0}\right)+\int_{\mathbb{R}^{d}} u_{0}^{2} d \mu<0 .
$$

Assume (ii). Then there exists a $\psi_{0} \in C_{0}^{\infty}\left(\mathbb{R}^{d}\right)$ with $\int_{\mathbb{R}^{d}} \psi_{0}^{2} d x=1$ such that

$$
\mathcal{E}^{(\alpha)}\left(\psi_{0}, \psi_{0}\right)+\int_{\mathbb{R}^{d}} \psi_{0}^{2} d \mu<0 .
$$

Letting

we have

$$
u_{0}=\frac{\psi_{0}}{\sqrt{\int_{\mathbb{R}^{d}} \psi_{0}^{2} d \mu^{-}}},
$$

$$
\mathcal{E}^{(\alpha)}\left(u_{0}, u_{0}\right)+\int_{\mathbb{R}^{d}} u_{0}^{2} d \mu^{+}<1
$$


Remark 2.3. We see from [29, Lemma 3.5] that if

$$
\inf \left\{\mathcal{E}^{(\alpha)}(u, u)+\int_{\mathbb{R}^{d}} u^{2} d \mu: \int_{\mathbb{R}^{d}} u^{2} d x=1\right\}>0,
$$

then

$$
\inf \left\{\mathcal{E}^{(\alpha)}(u, u)+\int_{\mathbb{R}^{d}} u^{2} d \mu^{+}: \int_{\mathbb{R}^{d}} u^{2} d \mu^{-}=1\right\}>1 .
$$

However, the converse does not hold in general. In fact, let $\alpha=2$ and $\mu=-\sigma_{R}$, the surface measure of the sphere $\partial B(R)$. Then if $R<\frac{d-2}{2}$, the first infimum is equal to zero, while the second one is greater than $1([29])$.

Define

$$
\begin{aligned}
& \lambda^{+}=\inf \{\lambda>0: C(\lambda)>0\}, \\
& \lambda^{-}=\sup \{\lambda<0: C(\lambda)>0\} .
\end{aligned}
$$

Lemma 2.4. Let $\mu=\mu^{+}-\mu^{-} \in \mathcal{K}_{d, \alpha}^{\infty}-\mathcal{K}_{d, \alpha}^{\infty}$ with $\mu^{-} \not \equiv 0$ (resp. $\left.\mu^{+} \not \equiv 0\right)$. Then the number $\lambda^{+}$(resp. $\left.\lambda^{-}\right)$is characterized as a unique positive (resp. negative) number such that

$$
\begin{gathered}
\inf \left\{\mathcal{E}^{(\alpha)}(u, u)+\lambda^{+} \int_{\mathbb{R}^{d}} u^{2} d \mu^{+}: \lambda^{+} \int_{\mathbb{R}^{d}} u^{2} d \mu^{-}=1\right\}=1 \\
\left(\text { resp. } \inf \left\{\mathcal{E}^{(\alpha)}(u, u)-\lambda^{-} \int_{\mathbb{R}^{d}} u^{2} d \mu^{-}:-\lambda^{-} \int_{\mathbb{R}^{d}} u^{2} d \mu^{+}=1\right\}=1\right) .
\end{gathered}
$$

Remark 2.5. From now on, we suppose that $\mu^{-}$is non-trivial, $\mu^{-} \not \equiv 0$, and consider the differentiability of $C(\lambda)$ at $\lambda=\lambda^{+}$. The proof for other cases is precisely the same. We know from [30, Lemma 4.2] that $\lambda^{+}>0$.

Proof of Lemma 2.4. Let $\mathbb{R}^{d}=F+F^{c}$ be the Hahn decomposition: $\mu^{-}(F)=$ $\mu^{-}\left(\mathbb{R}^{d}\right), \mu^{+}\left(F^{c}\right)=\mu^{+}\left(\mathbb{R}^{d}\right)$. Take $R>0$ so large that $\mu^{-}(F \cap B(R))>0$ and let $A=F \cap B(R)$. Take a sequence of non-negative functions $f_{n}$ in $C_{0}^{\infty}\left(\mathbb{R}^{d}\right)$ such that

$$
\int_{\mathbb{R}^{d}}\left(I_{A}(x)-f_{n}(x)\right)^{2}|\mu|(d x) \longrightarrow 0 \quad \text { as } n \rightarrow \infty .
$$

It then holds that

$$
\lim _{n \rightarrow \infty} \int_{\mathbb{R}^{d}} f_{n}^{2}(x) \mu^{-}(d x)=\mu^{-}(A)>0, \quad \lim _{n \rightarrow \infty} \int_{\mathbb{R}^{d}} f_{n}^{2}(x) \mu^{+}(d x)=\mu^{+}(A)=0,
$$

and consequently, there exists a function $f \in C_{0}^{\infty}\left(\mathbb{R}^{d}\right)$ such that

$$
\int_{\mathbb{R}^{d}} f^{2}(x) \mu^{-}(d x)=1, \quad \int_{\mathbb{R}^{d}} f^{2}(x) \mu^{+}(d x)<1 .
$$

Set

$$
F(\lambda)=\inf \left\{\mathcal{E}^{(\alpha)}(u, u)+\lambda \int_{\mathbb{R}^{d}} u^{2}(x) \mu^{+}(d x): \int_{\mathbb{R}^{d}} u^{2}(x) \mu^{-}(d x)=1\right\} .
$$

First we find that $F(0)>0(F(0)$ is the bottom of the spectrum of the time changed process of $\mathbf{M}^{\alpha}$ by the additive functional $A_{t}^{\mu^{-}}$([27, Lemma 3.1])). Indeed, since the embedding of $\mathcal{D}_{e}\left(\mathcal{E}^{(\alpha)}\right)$ to $L^{2}\left(\mu^{-}\right)$is compact by Theorem 3.4 below, there exists the function $u_{0}$ in $\mathcal{D}_{e}\left(\mathcal{E}^{(\alpha)}\right)$ that attains the infimum of (2.9). If $F(0)=0$, then $\mathcal{E}^{(\alpha)}\left(u_{0}, u_{0}\right)=0$, and thus $u_{0}=0$ because $\left(\mathcal{D}_{e}\left(\mathcal{E}^{(\alpha)}\right), \mathcal{E}^{(\alpha)}\right)$ is a Hilbert space. This contradicts that $\int_{\mathbb{R}^{d}} u_{0}^{2} d \mu^{-}=1$. Moreover, $F(\lambda), \lambda \geq 0$, is a concave function by the 
definition and dominated by the function $G(\lambda):=\mathcal{E}^{(\alpha)}(f, f)+\lambda \int_{\mathbb{R}^{d}} f^{2}(x) \mu^{+}(d x)$, where $f$ is a function satisfying (2.8).

These properties of $F$ show that there exists a unique $\lambda^{0}>0$ such that $F\left(\lambda^{0}\right)=$ $\lambda^{0}$. We see from Lemma 2.2 that $\lambda^{0}=\lambda^{+}$and thus $F\left(\lambda^{+}\right) / \lambda^{+}=1$, which leads us to the lemma.

The operator $\mathcal{H}^{\mu}$ is said to be subcritical, if $\mathcal{H}^{\mu}$ possesses the minimal positive Green function $G^{\mu}(x, y)$, that is,

$$
G^{\mu}(x, y)=\int_{0}^{\infty} p^{\mu}(t, x, y) d t<\infty, \quad x \neq y .
$$

It was shown in 32 that the following condition is a necessary and sufficient one for a operator $\mathcal{H}^{\mu}$ being subcritical:

$$
\inf \left\{\mathcal{E}^{(\alpha)}(u, u)+\int_{\mathbb{R}^{d}} u^{2} d \mu^{+}: \int_{\mathbb{R}^{d}} u^{2} d \mu^{-}=1\right\}>1 .
$$

Lemma 2.4 proves that operators $\mathcal{H}^{\lambda^{+} \mu}$ and $\mathcal{H}^{\lambda^{-}} \mu$ are not subcritical.

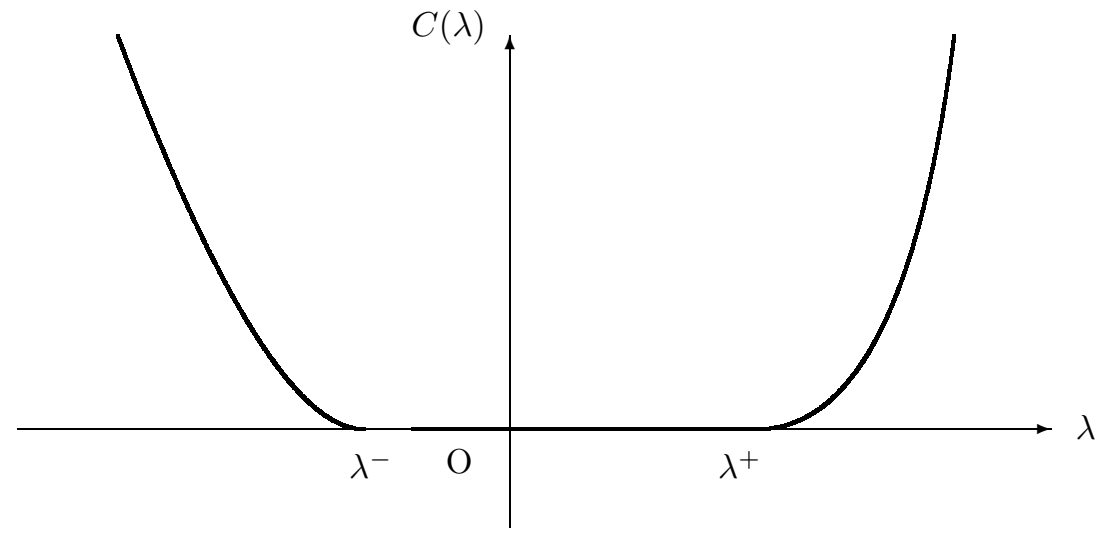

Figure. Spectral function

3. Compact embedding of $\mathcal{D}_{e}\left(\mathcal{E}^{(\alpha)}\right)$ into $L^{2}(\mu)$

In this section, we prove that for $\mu \in \mathcal{K}_{d, \alpha}^{\infty}$ the embedding of $\mathcal{D}_{e}\left(\mathcal{E}^{(\alpha)}\right)$ into $L^{2}(\mu)$ is compact.

Lemma 3.1. Let $\varphi \in C_{0}^{\infty}\left(\mathbb{R}^{d}\right)$ and $u \in \mathcal{D}_{e}\left(\mathcal{E}^{(\alpha)}\right)$. Then $u \varphi \in \mathcal{D}_{e}\left(\mathcal{E}^{(\alpha)}\right)$, and there exists a constant $C$ depending only on $\varphi$ such that

$$
\mathcal{E}^{(\alpha)}(u \varphi, u \varphi) \leq C \mathcal{E}^{(\alpha)}(u, u)
$$


Proof. By the inequality " $(a+b)^{2} \leq 2\left(a^{2}+b^{2}\right)$ ",

$$
\begin{aligned}
& \iint_{\mathbb{R}^{d} \times \mathbb{R}^{d} \backslash d} \frac{(u(x) \varphi(x)-u(y) \varphi(y))^{2}}{|x-y|^{d+\alpha}} d x d y \\
\leq & \iint_{\mathbb{R}^{d} \times \mathbb{R}^{d} \backslash d} \frac{u(x)^{2}(\varphi(x)-\varphi(y))^{2}}{|x-y|^{d+\alpha}} d x d y+\iint_{\mathbb{R}^{d} \times \mathbb{R}^{d} \backslash d} \frac{\varphi(y)^{2}(u(x)-u(y))^{2}}{|x-y|^{d+\alpha}} d x d y \\
= & (\mathrm{I})+(\mathrm{II}) .
\end{aligned}
$$

By Hölder's inequality, the first term (I) is dominated by

$$
\left(\iint_{\mathbb{R}^{d} \times \mathbb{R}^{d} \backslash d}|u(x)|^{p} \frac{(\varphi(x)-\varphi(y))^{2}}{|x-y|^{d+\alpha}} d x d y\right)^{\frac{2}{p}}\left(\iint_{\mathbb{R}^{d} \times \mathbb{R}^{d} \backslash d} \frac{(\varphi(x)-\varphi(y))^{2}}{|x-y|^{d+\alpha}} d x d y\right)^{\frac{p-2}{p}},
$$

where $1 / p=1 / 2-\alpha / 2 d$. We put

$$
(\mathrm{III})=\iint_{\mathbb{R}^{d} \times \mathbb{R}^{d} \backslash d}|u(x)|^{p} \frac{(\varphi(x)-\varphi(y))^{2}}{|x-y|^{d+\alpha}} d x d y,
$$

and divide (III) as a sum of two terms

$$
\iint_{|x-y| \leq 1}|u(x)|^{p} \frac{(\varphi(x)-\varphi(y))^{2}}{|x-y|^{d+\alpha}} d x d y+\iint_{|x-y|>1}|u(x)|^{p} \frac{(\varphi(x)-\varphi(y))^{2}}{|x-y|^{d+\alpha}} d x d y .
$$

Since $|\varphi(x)-\varphi(y)|^{2} \leq c|x-y|^{2}$, the first term of (3.2) is bounded by

$$
\begin{aligned}
& c \iint_{|x-y| \leq 1}|u(x)|^{p} \frac{1}{|x-y|^{d+\alpha-2}} d x d y \\
= & C\|u\|_{p}^{p} \int_{0}^{1} \frac{r^{d-1}}{r^{d+\alpha-2}} d r=C\|u\|_{p}^{p} .
\end{aligned}
$$

The second term of (3.2) is bounded by

$$
4 C\|\varphi\|_{\infty}^{2}\|u\|_{p}^{p} \int_{1}^{\infty} \frac{r^{d-1}}{r^{d+\alpha}} d r \leq C\|u\|_{p}^{p}
$$

Hence (III) $)^{2 / p}$ is bounded by $C\|u\|_{p}^{2}$. Using the Sobolev inequality for order $\alpha / 2$ ([12, p.44, (1.5.20)]), the first term (I) is bounded by $C \mathcal{E}^{(\alpha)}(u, u)$. Since the second term (II) is also bounded by $C\|\varphi\|_{\infty}^{2} \mathcal{E}^{(\alpha)}(u, u)$, we see from the definition of $\mathcal{D}\left(\mathcal{E}^{(\alpha)}\right)$ that the for $u \in \mathcal{D}\left(\mathcal{E}^{(\alpha)}\right)$, the product $u \varphi$ belongs to $\mathcal{D}\left(\mathcal{E}^{(\alpha)}\right)$ and the inequality (3.1) holds. This can be extended to $u \in \mathcal{D}_{e}\left(\mathcal{E}^{(\alpha)}\right)$.

Now we provide known facts on measures in the Kato class. Let $G_{\beta}(x, y)$ be the $\beta$-resolvent kernel of $\mathbf{M}^{\alpha}$.

Theorem $3.2([25])$. Let $\mu \in \mathcal{K}_{d, \alpha}$. Then

$$
\int_{\mathbb{R}^{d}} u^{2}(x) \mu(d x) \leq\left\|G_{\beta} \mu\right\|_{\infty} \mathcal{E}_{\beta}^{(\alpha)}(u, u), \quad u \in \mathcal{D}\left(\mathcal{E}^{(\alpha)}\right),
$$

where $\mathcal{E}_{\beta}^{(\alpha)}(u, u)=\mathcal{E}^{(\alpha)}(u, u)+\beta \int_{\mathbb{R}^{d}} u^{2} d x$.

It is known from [1] (see also [34) that $\mu \in \mathcal{K}_{d, \alpha}$ if and only if

$$
\lim _{\beta \rightarrow \infty}\left\|G_{\beta} \mu\right\|_{\infty}=0 \text {. }
$$


Therefore the equation (3.3) shows that for any $\epsilon>0$ there exists $M(\epsilon)$ such that

$$
\int_{\mathbb{R}^{d}} u^{2}(x) \mu(d x) \leq \epsilon \mathcal{E}^{(\alpha)}(u, u)+M(\epsilon) \int_{\mathbb{R}^{d}} u^{2} d x, \quad u \in \mathcal{D}\left(\mathcal{E}^{(\alpha)}\right) .
$$

It is known in [7, Proposition 2.2] that $\mu \in \mathcal{K}_{d, \alpha}^{\infty}$ is Green bounded:

$$
\sup _{x \in \mathbb{R}^{d}} G \mu(x)=\sup _{x \in \mathbb{R}^{d}} E_{x}\left[A_{\infty}^{\mu}\right]<\infty .
$$

Hence the equation (3.5) says that for $\mu \in \mathcal{K}_{d, \alpha}^{\infty}$

$$
\int_{\mathbb{R}^{d}} u^{2} d \mu \leq\|G \mu\|_{\infty} \mathcal{E}^{(\alpha)}(u, u), \quad u \in \mathcal{D}_{e}\left(\mathcal{E}^{(\alpha)}\right),
$$

in particular, $L^{2}(\mu)$ is contained in $\mathcal{D}_{e}\left(\mathcal{E}^{(\alpha)}\right)$.

Recall that the extended Dirichlet space $\mathcal{D}_{e}\left(\mathcal{E}^{(\alpha)}\right)$ is a Hilbert space with inner product $\mathcal{E}^{(\alpha)}$.

Lemma 3.3. Let $u_{n} \in \mathcal{D}_{e}\left(\mathcal{E}^{(\alpha)}\right), n=1,2, \ldots$, be a sequence such that $u_{n}$ converges to $u$ weakly in $\mathcal{D}_{e}\left(\mathcal{E}^{(\alpha)}\right)$. Then for any set $A$ of finite Lebesgue measure, $u_{n} I_{A}$ converges to $u I_{A}$ strongly in $L^{2}(m)$.

Proof. The proof of this lemma is just the argument in [14, Theorem 8.6].

First note that the semigroup $P_{t}$ of $\mathbf{M}^{\alpha}$ can be uniquely extended to a linear operator on $\mathcal{D}_{e}\left(\mathcal{E}^{(\alpha)}\right)$ and that

$$
\left\|u-P_{t} u\right\|_{2} \leq \sqrt{t} \mathcal{E}^{(\alpha)}(u, u)^{1 / 2}, \quad u \in \mathcal{D}_{e}\left(\mathcal{E}^{(\alpha)}\right)
$$

(see [12, Lemma 1.5.4]). We then have

$$
\begin{aligned}
\left\|\left(u_{n}-u\right) I_{A}\right\|_{2} & \leq\left\|\left(u_{n}-P_{t} u_{n}\right) I_{A}\right\|_{2}+\left\|\left(P_{t} u_{n}-P_{t} u\right) I_{A}\right\|_{2}+\left\|\left(P_{t} u-u\right) I_{A}\right\|_{2} \\
& \leq 2 \sqrt{t} \sup _{n} \mathcal{E}^{(\alpha)}\left(u_{n}, u_{n}\right)+\left\|\left(P_{t} u_{n}-P_{t} u\right) I_{A}\right\|_{2} .
\end{aligned}
$$

By the Sobolev inequality, $u_{n}$ is a bounded sequence in $L^{p}(m), 1 / p=1 / 2-\alpha / 2 d$ and thus there exists an $L^{p}(m)$-weakly convergent subsequence. Using the BanachSaks Theorem, as in the proof of [12, Lemma 3.2.2], we can show that the entire sequence $u_{n}$ converges to $u$ weakly in $L^{p}(m)$. Using the Sobolev inequality again, we see that the integral kernel $p_{t}(x, y)$ of $P_{t}$ is bounded. Consequently, $p_{t}(x, \cdot) \in L^{q}(m)$ $(1 / q+1 / p=1),\left\|P_{t} u_{n}\right\|_{\infty}$ is bounded in $n$, and $P_{t} u_{n}$ converges to $P_{t} u$ m-a.e. Hence, by the dominated convergence theorem the last term of (3.8) converges to zero as $n \rightarrow \infty$. This lemma follows by letting $n \rightarrow \infty$ and $t \rightarrow 0$ in (3.8).

For a measure $\mu$, let us denote

$$
\mu_{R}(\cdot)=\mu(\cdot \cap B(R)), \quad \mu_{R^{c}}=\mu\left(\cdot \cap B(R)^{c}\right) .
$$

Theorem 3.4. If $\mu \in \mathcal{K}_{d, \alpha}^{\infty}$, then the embedding of $\mathcal{D}_{e}\left(\mathcal{E}^{(\alpha)}\right)$ into $L^{2}(\mu)$ is compact.

Proof. First note that the embedding of $\mathcal{D}_{e}\left(\mathcal{E}^{(\alpha)}\right)$ into $L^{2}(\mu)$ is bounded by (3.7). Let $\left\{u_{n}\right\}$ be a sequence in $\mathcal{D}_{e}\left(\mathcal{E}^{(\alpha)}\right)$ such that $u_{n} \rightarrow u$ weakly in $\mathcal{D}_{e}\left(\mathcal{E}^{(\alpha)}\right)$. Then Lemma 3.3 says that for $R>0$

$$
u_{n} I_{B(R)} \rightarrow u I_{B(R)} \quad L^{2}(m) \text {-strongly. }
$$


Now fix a function $\varphi \in C_{0}^{\infty}\left(\mathbb{R}^{d}\right)$ with $\varphi=1$ on $B(R)$. Then by (3.5)

$$
\begin{aligned}
\int_{\mathbb{R}^{d}}\left|u_{n}-u\right|^{2} \mu_{R}(d x)=\int_{\mathbb{R}^{d}}\left|u_{n} \varphi-u \varphi\right|^{2} \mu_{R}(d x) \\
\quad \leq \epsilon \mathcal{E}^{(\alpha)}\left(u_{n} \varphi-u \varphi, u_{n} \varphi-u \varphi\right)+M(\epsilon) \int_{\mathbb{R}^{d}}\left|u_{n} \varphi-u \varphi\right|^{2} d x .
\end{aligned}
$$

The second term of the right hand side converges to 0 as $n \rightarrow \infty$ by (3.9), and Lemma 3.1 proves

$$
\sup _{n} \mathcal{E}^{(\alpha)}\left(u_{n} \varphi-u \varphi, u_{n} \varphi-u \varphi\right)<\infty .
$$

Hence the sequence $\left\{u_{n}\right\}$ is $L^{2}\left(\mu_{R}\right)$-convergent to $u$ because $\epsilon$ is arbitrary.

Moreover, since by Theorem 3.2 ,

$$
\begin{gathered}
\int_{\mathbb{R}^{d}}\left|u_{n}-u\right|^{2} \mu(d x)=\int_{\mathbb{R}^{d}}\left|u_{n}-u\right|^{2} \mu_{R}(d x)+\int_{\mathbb{R}^{d}}\left|u_{n}-u\right|^{2} \mu_{R^{c}}(d x) \\
\leq \int_{\mathbb{R}^{d}}\left|u_{n}-u\right|^{2} \mu_{R}(d x)+\left\|G \mu_{R^{c}}\right\|_{\infty} \mathcal{E}^{(\alpha)}\left(u_{n}-u, u_{n}-u\right),
\end{gathered}
$$

we have

$$
\limsup _{n \rightarrow \infty} \int_{\mathbb{R}^{d}}\left|u_{n}-u\right|^{2} \mu(d x) \leq \limsup _{n \rightarrow \infty}\left\|G \mu_{R^{c}}\right\|_{\infty} \mathcal{E}^{(\alpha)}\left(u_{n}-u, u_{n}-u\right) .
$$

By the definition of $\mathcal{K}_{d, \alpha}^{\infty}$ the right hand side converges to 0 as $R \rightarrow \infty$, which proves that $\left\{u_{n}\right\}$ is an $L^{2}(\mu)$-convergent sequence to $u$.

\section{Construction of ground states}

We define an $\mathcal{H}^{\mu}$-harmonic function probabilistically.

Definition 4.1. A bounded finely continuous function $h$ on $\mathbb{R}^{d}$ is said to be $\mathcal{H}^{\mu}$ harmonic, if for any relatively compact domain $D \subset \mathbb{R}^{d}$,

$$
h(x)=E_{x}\left[\exp \left(-A_{\tau_{D}}^{\mu}\right) h\left(X_{\tau_{D}}\right)\right], \quad x \in D,
$$

where $\tau_{D}$ is the first exit time from $D$.

If $\mathcal{H}^{\mu}$ is subcritical, the measure $\mu$ is gaugeable, that is, $\sup _{x \in \mathbb{R}^{d}} E_{x}\left[e^{-A_{\infty}^{\mu}}\right]<\infty$ ([32, Theorem 3.1]). Set $h(x)=\mathbb{E}_{x}\left[e^{-A_{\infty}^{\mu}}\right]$. Then $h$ is an $\mathcal{H}^{\mu}$-harmonic function. Indeed, for any bounded domain $D$

$$
\begin{aligned}
& h(x)=E_{x}\left[e^{-A_{\infty}^{\nu}} h\left(X_{\tau_{D}}\right)\right]=E_{x}\left[e^{-A_{\tau_{D}}^{\nu}} E_{X_{\tau_{D}}}\left[e^{-A_{\infty}^{\nu}}\right]\right] \\
& =E_{x}\left[e^{\left.-A_{\tau_{D}}^{\nu} h\left(X_{\tau_{D}}\right)\right], \quad x \in D .}\right.
\end{aligned}
$$

Moreover, we see from (3.6) and Jensen's inequality that

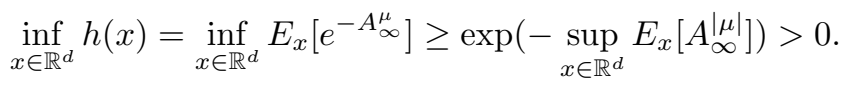

$\mathcal{H}^{\mu}$ is said to be critical, if $\mathcal{H}^{\mu}$ is not subcritical and admits a positive $\mathcal{H}^{\mu}$-harmonic function (cf. [19, p.145]). Recall that the operator $\mathcal{H}^{\lambda^{+} \mu}$ is not subcritical.

Let $P_{t}$ be a positive semigroup with integral kernel $p(t, x, y)$. A positive function $h$ is called $P_{t}$-excessive if $h$ satisfies $P_{t} h(x) \uparrow h(x)$ as $t \downarrow 0$. For a $P_{t}$-excessive function $h$, set

$$
p^{h}(t, x, y)=\frac{1}{h(x)} p(t, x, y) h(y), \quad t>0, x, y \in\{0<h<\infty\}
$$


and denote by $P_{t}^{h}$ the associated semigroup, $P_{t}^{h} f(x)=\int_{\{0<h<\infty\}} p^{h}(t, x, y) f(y) d y$. Then $p^{h}(t, x, y)$ becomes a transition probability density because

$$
P_{t}^{h} 1(x)=\frac{1}{h(x)} P_{t} h(x) \leq \frac{h(x)}{h(x)}=1 .
$$

We call the process generated by $p^{h}$ Doob's $h$-transformed process.

Lemma 4.2. A positive $\mathcal{H}^{\mu}$-harmonic function $h$ is $P_{t}^{\mu}$-excessive.

Proof. It follows from the definition of an $\mathcal{H}^{\mu}$-harmonic function that $\lim _{t \rightarrow 0} P_{t}^{\mu} h(x)$ $=h(x)$.

Let $x \in B(m)$. By Definition 4.1, $h$ satisfies

$$
h(x)=E_{x}\left[\exp \left(A_{\tau_{n}}^{\mu}\right) h\left(X_{\tau_{n}}\right)\right]
$$

for any $n>m$. Here $\tau_{n}$ is the first exit time from $B(n)$. It follows from the Markov property that

$$
\begin{aligned}
& E_{x}\left[\exp \left(A_{t}^{\mu}\right) h\left(X_{t}\right) ; t<\tau_{m}\right] \\
= & E_{x}\left[\exp \left(A_{t}^{\mu}\right) E_{X_{t}}\left[\exp \left(A_{\tau_{n}}^{\mu}\right) h\left(X_{\tau_{n}}\right)\right] ; t<\tau_{m}\right] \\
= & E_{x}\left[\exp \left(A_{t}^{\mu}\right) \exp \left(A_{\tau_{n}}^{\mu} \circ \theta_{t}\right) h\left(X_{\tau_{n}} \circ \theta_{t}\right) ; t<\tau_{m}\right] \\
= & E_{x}\left[\exp \left(A_{\tau_{n}}^{\mu}\right) h\left(X_{\tau_{n}}\right) ; t<\tau_{m}\right] \leq h(x) .
\end{aligned}
$$

Hence we have

$$
P_{t}^{\mu} h(x)=\lim _{m \rightarrow \infty} E_{x}\left[\exp \left(A_{t}^{\mu}\right) h\left(X_{t}\right) ; t<\tau_{m}\right] \leq h(x) .
$$

Assume that $\mathcal{H}^{\mu}$ is subcritical or critical and let $h$ be a positive $\mathcal{H}^{\mu}$-harmonic function. Let $P_{t}^{\mu, h}$ be the $h$-transformed semigroup of $P_{t}^{\mu}$. Then, $P_{t}^{\mu, h}$ is the $L^{2}\left(h^{2} m\right)$-strongly continuous symmetric Markov semigroup, $\left(P_{t}^{\mu, h} f, g\right)_{h^{2} m}=$ $\left(f, P_{t}^{\mu, h} g\right)_{h^{2} m}$. Denote by $\left(\mathcal{E}^{\mu, h}, \mathcal{D}\left(\mathcal{E}^{\mu, h}\right)\right)$ the Dirichlet space on $L^{2}\left(h^{2} m\right)$ generated by $P_{t}^{\mu, h}$. We define a symmetric positive form $\left(\mathcal{E}^{\mu}, \mathcal{D}_{e}\left(\mathcal{E}^{\mu}\right)\right)$ by

$$
\begin{aligned}
\mathcal{E}^{\mu}(u, v) & =\mathcal{E}^{\mu, h}\left(\frac{u}{h}, \frac{v}{h}\right), \\
\mathcal{D}_{e}\left(\mathcal{E}^{\mu}\right) & =\left\{u: \frac{u}{h} \in \mathcal{D}_{e}\left(\mathcal{E}^{\mu, h}\right)\right\} .
\end{aligned}
$$

We call the form $\left(\mathcal{E}^{\mu}, \mathcal{D}_{e}\left(\mathcal{E}^{\mu}\right)\right)$ an extended Schrödinger form. By the definition of $\mathcal{D}_{e}\left(\mathcal{E}^{\mu, h}\right)$ ([12, p.35]), $\mathcal{D}_{e}\left(\mathcal{E}^{\mu}\right)$ is identified with the family of an $m$-measurable function $u$ on $\mathbb{R}^{d}$ such that $|u|<\infty m$-a.e. and there exists an $\mathcal{E}^{\mu}$-Cauchy sequence $\left\{u_{n}\right\}$ of functions in $\mathcal{D}\left(\mathcal{E}^{(\alpha)}\right)$ such that $\lim _{n \rightarrow \infty} u_{n}=u m$-a.e. We call $\left\{u_{n}\right\}$ as above an approximating sequence for $u \in \mathcal{D}_{e}\left(\mathcal{E}^{\mu}\right)$. Then, for $u \in \mathcal{D}_{e}\left(\mathcal{E}^{\mu}\right)$ and its approximating sequence $\left\{u_{n}\right\}$

$$
\mathcal{E}^{\mu}(u, u)=\lim _{n \rightarrow \infty} \mathcal{E}^{\mu}\left(u_{n}, u_{n}\right), \quad u \in \mathcal{D}_{e}\left(\mathcal{E}^{\mu}\right)
$$

Moreover, in the second definition of $\mathcal{D}_{e}\left(\mathcal{E}^{\mu}\right)$, the condition for $\left\{u_{n}\right\}$ being an $\mathcal{E}^{\mu}$ Cauchy sequence can be replaced by

$$
\sup _{n} \mathcal{E}^{\mu}\left(u_{n}, u_{n}\right)<\infty
$$

([23, Definition 1.6]). 
If $\left(\mathcal{E}^{\mu}, \mathcal{D}\left(\mathcal{E}^{(\alpha)}\right)\right)$ is a subcritical Schrödinger form, that is, the associated operator $\mathcal{H}^{\mu}$ is subcritical, then $\left(\mathcal{E}^{\mu}, \mathcal{D}_{e}\left(\mathcal{E}^{\mu}\right)\right)$ becomes a Hilbert space by [12, Lemma 1.5.5]. If $\left(\mathcal{E}^{\mu}, \mathcal{D}\left(\mathcal{E}^{(\alpha)}\right)\right)$ is a critical Schrödinger form, that is, the associated operator $\mathcal{H}^{\mu}$ is critical, its ground state $h$ belongs to $\mathcal{D}_{e}\left(\mathcal{E}^{\mu}\right)$ on account of [12, Theorem 1.6.3]. Noting that for $\mu=\mu^{+}-\mu^{-} \in \mathcal{K}_{d, \alpha}^{\infty}-\mathcal{K}_{d, \alpha}^{\infty}$

$$
\mathcal{E}^{\mu}(u, u) \leq\left(1+\|G|\mu|\|_{\infty}\right) \mathcal{E}^{(\alpha)}(u, u)
$$

by Theorem 3.2, we see that $\mathcal{D}_{e}\left(\mathcal{E}^{\mu}\right)$ includes $\mathcal{D}_{e}\left(\mathcal{E}^{(\alpha)}\right)$.

For a non-negative bounded Borel function $w \not \equiv 0$ with compact support, define $\nu=\lambda^{+} \mu+w d x$. We then see from Lemma 2.4 and [32, Theorem 3.1] that $\mathcal{H}^{\nu}$ is subcritical and its Green function $G^{\nu}(x, y)$ is equivalent to $G$ : there exist positive constants $c, C$ such that

$$
c G(x, y) \leq G^{\nu}(x, y) \leq C G(x, y) \text { for } x \neq y .
$$

Let $G^{\nu}$ be the Green operator, $G^{\nu} f(x)=\int_{\mathbb{R}^{d}} G^{\nu}(x, y) f(y) d y$.

Lemma 4.3. For a non-negative function $\varphi \in C_{0}\left(\mathbb{R}^{d}\right), G^{\nu} \varphi$ belongs to $\mathcal{D}_{e}\left(\mathcal{E}^{\nu}\right)$.

Proof. Let $G_{\beta}^{\nu}$ be $\beta$-resolvent of $\mathcal{H}^{\nu}$. Then $G_{\beta}^{\nu} \varphi$ belongs to $\mathcal{D}\left(\mathcal{E}^{(\alpha)}\right)$ and $G_{\beta}^{\nu} \varphi \uparrow G^{\nu} \varphi$ as $\beta \rightarrow 0$. Moreover, by (4.5)

$$
\begin{aligned}
\mathcal{E}^{\nu}\left(G_{\beta}^{\nu} \varphi, G_{\beta}^{\nu} \varphi\right) & \leq \mathcal{E}_{\beta}^{\nu}\left(G_{\beta}^{\nu} \varphi, G_{\beta}^{\nu} \varphi\right)=\left(\varphi, G_{\beta}^{\nu} \varphi\right) \\
& \leq\left(\varphi, G^{\nu} \varphi\right) \leq C(\varphi, G \varphi)<\infty
\end{aligned}
$$

which proves the lemma.

Noting that $\nu$ is gaugeable, we set $h_{0}(x)=\mathbb{E}_{x}\left[e^{-A_{\infty}^{\nu}}\right]$. Then $h_{0}$ is an $\mathcal{H}^{\nu}$-harmonic function as mentioned above. Let $\left(\mathcal{E}^{\nu, h_{0}}, \mathcal{D}\left(\mathcal{E}^{\nu, h_{0}}\right)\right)$ be the Dirichlet form generated by $h$-transformed semigroup $P_{t}^{\nu, h_{0}}$ and $G^{\nu, h_{0}}$ its Green operator :

$$
G^{\nu, h_{0}} f=\frac{1}{h_{0}} G^{\nu}\left(h_{0} \cdot f\right) .
$$

Then, for a non-negative function $\varphi \in C_{0}\left(\mathbb{R}^{d}\right)$

$$
\int_{\mathbb{R}^{d}} G^{\nu, h_{0}}\left(\frac{\varphi}{h_{0}}\right) \cdot \frac{\varphi}{h_{0}} h_{0}^{2} d x=\int_{\mathbb{R}^{d}} G^{\nu} \varphi \cdot \varphi d x \leq C \int_{\mathbb{R}^{d}} G \varphi \cdot \varphi d x .
$$

Thus Theorem 1.5.4 in [12] says that $\varphi / h_{0}$ belongs to $\mathcal{D}_{e}\left(\mathcal{E}^{\nu, h_{0}}\right)$, and for any $f \in$ $\mathcal{D}_{e}\left(\mathcal{E}^{\nu}\right)$

$$
\mathcal{E}^{\nu, h_{0}}\left(G^{\nu, h_{0}}\left(\frac{\varphi}{h_{0}}\right), \frac{f}{h_{0}}\right)=\int_{\mathbb{R}^{d}} \varphi f d x .
$$

Noting that the left hand side above equals $\mathcal{E}^{\nu}\left(G^{\nu} \varphi, f\right)$, we have

Lemma 4.4. For any non-negative function $\varphi \in C_{0}\left(\mathbb{R}^{d}\right)$

$$
\mathcal{E}^{\nu}\left(G^{\nu} \varphi, f\right)=\int_{\mathbb{R}^{d}} \varphi f d x, \quad f \in \mathcal{D}_{e}\left(\mathcal{E}^{\nu}\right)
$$

We now construct an $\mathcal{H}^{\lambda^{+}} \mu$-harmonic function. Since the embedding of $\mathcal{D}_{e}\left(\mathcal{E}^{(\alpha)}\right)$ to $L^{2}\left(\mu^{-}\right)$is compact, there exists a function $u_{0} \in \mathcal{D}_{e}\left(\mathcal{E}^{(\alpha)}\right)$ such that $u_{0}$ attains the infimum:

$$
\inf \left\{\mathcal{E}^{(\alpha)}(u, u)+\lambda^{+} \int_{\mathbb{R}^{d}} u^{2} d \mu^{+}: u \in \mathcal{D}_{e}\left(\mathcal{E}^{(\alpha)}\right), \lambda^{+} \int_{\mathbb{R}^{d}} u^{2} d \mu^{-}=1\right\}=1 .
$$


Let $\mathbf{M}^{\lambda^{+} \mu^{+}}$be the subprocess of $\mathbf{M}^{\alpha}$ by the multiplicative functional $\exp \left(-\lambda^{+} A_{t}^{\mu^{+}}\right)$. Then the function $u_{0}$ is the first eigenfunction corresponding to the generator of the time changed process of $\mathbf{M}^{\lambda^{+} \mu^{+}}$by $A_{t}^{\lambda^{+} \mu^{-}}$. The time changed process is irreducible because $\int_{\mathbb{R}^{d}} G^{\lambda^{+} \mu^{+}}(x, y) \mu^{-}(d y)>0$. Hence $u_{0}>0 \mu^{-}$-a.e by [9, Theorem 7.3].

Lemma 4.5. Let $u_{0}$ be the function in (4.6). Then the measure $u_{0} \mu^{-}$is of finite energy integral with respect to $\mathcal{E}^{\lambda^{+} \mu^{+}}$.

Proof. Let $f \in \mathcal{D}_{e}\left(\mathcal{E}^{(\alpha)}\right)$. Then

$$
\int_{\mathbb{R}^{d}} f(x) u_{0}(x) \mu^{-}(d x) \leq\left(\int_{\mathbb{R}^{d}} u_{0}^{2}(x) \mu^{-}(d x)\right)^{1 / 2}\left(\int_{\mathbb{R}^{d}} f^{2}(x) \mu^{-}(d x)\right)^{1 / 2},
$$

and the right hand side is dominated by

$$
C \mathcal{E}^{(\alpha)}(f, f)^{1 / 2} \leq C \mathcal{E}^{\lambda^{+} \mu^{+}}(f, f)^{1 / 2}
$$

by Theorem 3.2

The function $u_{0}$ is also characterized by the equation:

$$
\mathcal{E}^{(\alpha)}\left(u_{0}, f\right)+\lambda^{+} \int_{\mathbb{R}^{d}} u_{0} f d \mu^{+}=\lambda^{+} \int_{\mathbb{R}^{d}} u_{0} f d \mu^{-}, \quad \text { for all } f \in \mathcal{D}_{e}\left(\mathcal{E}^{(\alpha)}\right) .
$$

Hence we see from Lemma 4.5 that

$$
\mathcal{E}^{\lambda^{+} \mu^{+}}\left(u_{0}, f\right)=\lambda^{+} \int_{\mathbb{R}^{d}} u_{0} f d \mu^{-}=\mathcal{E}^{\lambda^{+} \mu^{+}}\left(\lambda^{+} G^{\lambda^{+} \mu^{+}}\left(u_{0} \mu^{-}\right), f\right),
$$

and so

$$
\begin{aligned}
u_{0}(x) & =\lambda^{+} \int_{\mathbb{R}^{d}} G^{\lambda^{+} \mu^{+}}(x, y) u_{0}(y) d \mu^{-}(d y) \\
& =E_{x}\left[\int_{0}^{\infty} \exp \left(-\lambda^{+} A_{t}^{\mu^{+}}\right) u_{0}\left(X_{t}\right) d A_{t}^{\lambda^{+} \mu^{-}}\right]>0, m \text {-a.e. }
\end{aligned}
$$

Now we set

$$
h(x)=E_{x}\left[\int_{0}^{\infty} \exp \left(-\lambda^{+} A_{t}^{\mu^{+}}\right) u_{0}\left(X_{t}\right) d A_{t}^{\lambda^{+} \mu^{-}}\right]
$$

and prove that the function $h$ is a bound continuous $\mathcal{H}^{\lambda^{+}} \mu$-harmonic function. We remark that $h$ is equal to $u_{0}$ q.e. and is strictly positive because $G^{\lambda^{+} \mu^{+}}(x, y)>0$.

Lemma 4.6. The function $h$ is finely continuous.

Proof. By the Markov property,

$$
\begin{aligned}
h\left(X_{s}\right)= & E_{X_{s}}\left[\int_{0}^{\infty} \exp \left(-\lambda^{+} A_{t}^{\mu^{+}}\right) u_{0}\left(X_{t}\right) d A_{t}^{\lambda^{+} \mu^{-}}\right] \\
= & E_{x}\left[\int_{0}^{\infty} \exp \left(-\lambda^{+} A_{t}^{\mu^{+}}\left(\theta_{s}\right)\right) u_{0}\left(X_{t+s}\right) d A_{t}^{\lambda^{+} \mu^{-}}\left(\theta_{s}\right) \mid \mathcal{F}_{s}\right] \\
= & \exp \left(\lambda^{+} A_{s}^{\mu^{+}}\right) E_{x}\left[\int_{0}^{\infty} \exp \left(-\lambda^{+} A_{t}^{\mu^{+}}\right) u_{0}\left(X_{t}\right) d A_{t}^{\lambda^{+} \mu^{-}} \mid \mathcal{F}_{s}\right] \\
& -\exp \left(\lambda^{+} A_{s}^{\mu^{+}}\right) \int_{0}^{s} \exp \left(-\lambda^{+} A_{t}^{\mu^{+}}\right) u_{0}\left(X_{t}\right) d A_{t}^{\lambda^{+} \mu^{-}} .
\end{aligned}
$$

Since the first term of right hand side is right continuous because of the right continuity of $\mathcal{F}_{s}$, we see that $h$ is finely continuous by [12, Theorem A.2.7]. 
Note that if $h(x)=u_{0}(x) m$-a.e., then $h(x)=u_{0}(x)$ q.e. by [12, Lemma 4.1.5]. Hence [12, Theorem 4.1.2] proves the next lemma.

Lemma 4.7. The function $h$ is strictly positive and satisfies

$$
h(x)=E_{x}\left[\int_{0}^{\infty} \exp \left(-\lambda^{+} A_{t}^{\mu^{+}}\right) h\left(X_{t}\right) d A_{t}^{\lambda^{+} \mu^{-}}\right]
$$

for all $x \in \mathbb{R}^{d}$.

The next theorem was first obtained by Murata [16, Theorem 2.2] when $\alpha=$ 2. Using a probabilistic argument, we extend the theorem to symmetric $\alpha$-stable processes.

Theorem 4.8. For $w \in C_{0}\left(\mathbb{R}^{d}\right)$ with $w \geq 0, w \not \equiv 0$, let $\nu=\lambda^{+} \mu+w d x$. The function $h$ defined in (4.8) satisfies

$$
h(x)=\int_{\mathbb{R}^{d}} G^{\nu}(x, y) h(y) w(y) d y .
$$

Proof. Note that $h$ satisfies (4.7) and for any non-negative $\varphi \in C_{0}\left(\mathbb{R}^{d}\right)$

$$
\mathcal{E}^{\nu}\left(h, G_{\beta}^{\nu} \varphi\right)=\int_{\mathbb{R}^{d}} h \cdot G_{\beta}^{\nu} \varphi \cdot w d x, \beta>0 .
$$

By Lemma 4.3 and Lemma 4.4 we obtain, by letting $\beta$ to 0

$$
\int_{\mathbb{R}^{d}} h(x) \varphi(x) d x=\int_{\mathbb{R}^{d}} h(x) w(x) G^{\nu} \varphi(x) d x=\int_{\mathbb{R}^{d}} G^{\nu}(h w)(x) \varphi(x) d x
$$

and thus

$$
h(x)=\int_{\mathbb{R}^{d}} G^{\nu}(x, y) h(y) w(y) d y, \quad m \text {-a.e. }
$$

by the same argument as in Lemma 4.6, in the above equation " $m$-a.e. $x$ " can be replaced by "any $x$ ".

Lemma 4.9. The function $h$ is bounded.

Proof. Since $h$ is finely continuous, we can find a compact set $K$ such that $h \leq c$ on $K$. Let $\nu=\mu+I_{K}(x) d x$. Note that $\nu$ belongs to $\mathcal{K}_{d, \alpha}^{\infty}-\mathcal{K}_{d, \alpha}^{\infty}$. Theorem 4.8 says that $h$ satisfies

$$
h(x)=\int_{\mathbb{R}^{d}} G^{\nu}(x, y) h(y) I_{K}(y) d y .
$$

Since $G^{\nu}(x, y)$ is equivalent to $G(x, y)$ by [32, Theorem 3.1], it holds that

$$
h(x) \leq c \int_{\mathbb{R}^{d}} G^{\nu}(x, y) I_{K}(y) d y \leq C^{\prime} \int_{\mathbb{R}^{d}} G(x, y) I_{K}(y) d y .
$$

The right hand side of the above inequality is bounded because $h(y) I_{K}(y) d y \in$ $\mathcal{K}_{d, \alpha}^{\infty}$.

Lemma 4.10. The function $h$ is ${P_{t}^{\lambda^{+}} \mu}^{\text {-excessive. }}$ 
Proof. Set $M_{t}=E_{x}\left[\int_{0}^{\infty} \exp \left(-\lambda^{+} A_{s}^{\mu^{+}}\right) h\left(X_{s}\right) d A_{s}^{\lambda^{+} \mu^{-}} \mid \mathcal{F}_{t}\right]$. Then we showed in the proof of Lemma 4.4 that

$$
\exp \left(-\lambda^{+} A_{t}^{\mu^{+}}\right) h\left(X_{t}\right)=M_{t}-\int_{0}^{t} \exp \left(-\lambda^{+} A_{s}^{\mu^{+}}\right) u_{0}\left(X_{s}\right) d A_{s}^{\lambda^{+} \mu^{-}} .
$$

Hence by Ito's formula

$$
\begin{aligned}
\exp ( & \left.-\lambda^{+} A_{t}^{\mu}\right) h\left(X_{t}\right)=\exp \left(\lambda^{+} A_{t}^{\mu^{-}}\right)\left(\exp \left(-\lambda^{+} A_{t}^{\mu^{+}}\right) h\left(X_{t}\right)\right) \\
= & h\left(X_{0}\right)+\int_{0}^{t} \exp \left(\lambda^{+} A_{s}^{\mu^{-}}\right) d M_{s}-\int_{0}^{t} \exp \left(-\lambda^{+} A_{s}^{\mu}\right) h\left(X_{s}\right) d A_{s}^{\lambda^{+} \mu^{-}} \\
& +\int_{0}^{t} \exp \left(-\lambda^{+} A_{s}^{\mu^{+}}\right) h\left(X_{s}\right) \exp \left(\lambda^{+} A_{s}^{\mu^{-}}\right) d A_{s}^{\lambda^{+} \mu^{-}} \\
= & h\left(X_{0}\right)+\int_{0}^{t} \exp \left(\lambda^{+} A_{s}^{\mu^{-}}\right) d M_{s}
\end{aligned}
$$

which implies that

$$
E_{x}\left[\exp \left(-\lambda^{+} A_{t}^{\mu}\right) h\left(X_{t}\right)\right] \leq h(x)
$$

We see from Lemma 4.10 that the $h$-transformed semigroup $P_{t}^{\lambda^{+} \mu, h}$ generates an $h^{2} m$-symmetric Markov process. Let us denote by $\mathbf{M}^{\lambda^{+}} \mu, h$ the Markov process generated by $P_{t}^{\lambda^{+} \mu, h}$. Then $\mathbf{M}^{\lambda^{+} \mu, h}$ is recurrent because of non-subcriticality of $\mathcal{H}^{\lambda^{+} \mu}$, in particular, conservative, $P_{t}^{\lambda^{+} \mu, h} 1=1$. As a result, the function $h$ is $P_{t}^{\lambda^{+} \mu}$-invariant:

$$
P_{t}^{\lambda^{+} \mu} h=h
$$

Lemma 4.11. A finely continuous $P_{t}^{\lambda^{+} \mu}$-excessive function is unique up to constant multiplication.

Proof. We follow the argument in [19, Theorem 3.4]. Let $h, h^{\prime}$ be finely continuous $P_{t}^{\lambda^{+} \mu}$-excessive functions. Since

$$
E_{x}\left[\exp \left(-\lambda^{+} A_{t}^{\mu}\right) h\left(X_{t}\right)\left(\frac{h^{\prime}}{h}\right)\left(X_{t}\right)\right] \leq h \cdot \frac{h^{\prime}}{h}(x),
$$

we have

$$
E_{x}^{\lambda^{+} \mu, h}\left[\frac{h^{\prime}}{h}\left(X_{t}\right)\right] \leq \frac{h^{\prime}}{h}(x)
$$

For $y \in \mathbb{R}^{d}$ and $\epsilon_{n} \rightarrow 0$ as $n \rightarrow \infty, \sigma_{B\left(y, \epsilon_{n}\right)}<\infty, P_{x}^{\lambda^{+} \mu, h}$-a.s by [12, Problem 4.6.3], where $B\left(y, \epsilon_{n}\right)=\left\{z:|z-y|<\epsilon_{n}\right\}$. Denote $\sigma_{n}=\sigma_{B\left(y, \epsilon_{n}\right)}$. Replacing $t$ by $\sigma_{n}$, we have

$$
E_{x}^{\lambda^{+} \mu, h}\left[\frac{h^{\prime}}{h}\left(X_{\sigma_{n}}\right)\right] \leq \frac{h^{\prime}}{h}(x)
$$


Noting that the left hand side of (4.12) converges to $\frac{h^{\prime}}{h}(y)$ as $n \rightarrow \infty$, we obtain by Fatou's lemma that

$$
\begin{aligned}
\frac{h^{\prime}}{h}(y) & =E_{x}^{\lambda^{+} \mu, h}\left[\liminf _{n \rightarrow \infty} \frac{h^{\prime}}{h}\left(X_{\sigma_{n}}\right)\right] \\
& \leq \liminf _{n \rightarrow \infty} E_{x}^{\lambda^{+} \mu, h}\left[\frac{h^{\prime}}{h}\left(X_{\sigma_{n}}\right)\right] \\
& \leq \frac{h^{\prime}}{h}(x) .
\end{aligned}
$$

Since $x$ and $y$ are arbitrary, $h^{\prime} / h$ must be a constant function.

Proposition 4.12. The function $h$ is an $\mathcal{H}^{\lambda^{+}} \mu_{\text {-harmonic function: for any bounded }}$ domain $D$

$$
E_{x}\left[\exp \left(-\lambda^{+} A_{\tau_{D}}^{\mu}\right) h\left(X_{\tau_{D}}\right)\right]=h(x), x \in D
$$

Proof. We first note that if the equation (4.13) holds for $D$, then it holds for any $D^{\prime} \subset D$ by the strong Markov property. Thus it suffices to prove this proposition for a large $D$ such that $\mu^{-}(D)>0$.

In Lemma4.6 and Lemma 4.9, we proved that $h$ is finely continuous and bounded. Set

$$
M_{t}=\exp \left(-\lambda^{+} A_{t}^{\mu}\right) h\left(X_{t}\right) .
$$

Then $M_{t}$ is a martingale. In fact, by the Markov property and the equation (4.11)

$$
\begin{aligned}
E_{x}\left[M_{t} \mid \mathcal{F}_{s}\right] & =\exp \left(-\lambda^{+} A_{s}^{\mu}\right) E_{X_{s}}\left[\exp \left(-\lambda^{+} A_{t-s}^{\mu}\right) h\left(X_{t-s}\right)\right] \\
& =\exp \left(-\lambda^{+} A_{s}^{\mu}\right) h\left(X_{s}\right), \quad t>s .
\end{aligned}
$$

On account of the optional stopping theorem, we have

$$
E_{x}\left[\exp \left(-\lambda^{+} A_{t \wedge \tau_{D}}\right) h\left(X_{t \wedge \tau_{D}}\right)\right]=h(x),
$$

where $D$ is a bounded domain of $\mathbb{R}^{d}$.

We claim that

$$
\inf \left\{\mathcal{E}^{\lambda^{+} \mu}(u, u): u \in \mathcal{D}\left(\mathcal{E}_{D}^{(\alpha)}\right), \int_{D} u^{2} d x=1\right\}>0,
$$

where $\mathcal{E}_{D}^{(\alpha)}$ is the part Dirichlet form of $\mathcal{E}^{(\alpha)}$ on $D$ (see [12, Section 4.4]). Indeed, if the left hand side equals zero, there exists a function $u_{*} \in \mathcal{D}\left(\mathcal{E}_{D}^{(\alpha)}\right)$ such that

$$
\mathcal{E}^{(\alpha)}\left(u_{*}, u_{*}\right)+\lambda^{+} \int_{\mathbb{R}^{d}} u_{*}^{2} d \mu^{+}=\lambda^{+} \int_{\mathbb{R}^{d}} u_{*}^{2} d \mu^{-} .
$$

Note that $\int_{\mathbb{R}^{d}} u_{*}^{2} d \mu^{-}>0$ because $u_{*}(x)>0$ q.e. on $D$ and $m^{-}(D)>0$. Then the function $u_{*} / \sqrt{\int_{\mathbb{R}^{d}} u_{*}^{2} d \mu^{-}}$attains the infimum of (4.6), and thus $u_{*}>0$ q.e. on $\mathbb{R}^{d}$ as proved for the function $u_{0}$. This is contradictory, because $u_{*}=0 \mathrm{~m}$-a.e. on $\mathbb{R}^{d} \backslash D$.

The equation (4.15) implies that $\lambda^{+} \mu$ is gaugeable on $D$, that is,

$$
\sup _{x \in D} E_{x}\left[\exp \left(-\lambda^{+} A_{\tau_{D}}\right)\right]<\infty
$$


(cf. [7, 32]). We then see from [7, Corollary 2.9] that

$$
\sup _{x \in D} E_{x}\left[\sup _{0 \leq t \leq \tau_{D}} \exp \left(-\lambda^{+} A_{t}^{\mu}\right)\right]<\infty
$$

Noting that

$$
\left|\exp \left(-\lambda^{+} A_{t \wedge \tau_{D}}\right) h\left(X_{t \wedge \tau_{D}}\right)\right| \leq\|h\|_{\infty}\left(\sup _{0 \leq t \leq \tau_{D}} \exp \left(-\lambda^{+} A_{t}^{\mu}\right)\right),
$$

we have

$$
\lim _{t \rightarrow \infty} E_{x}\left[\exp \left(-\lambda^{+} A_{t \wedge \tau_{D}}^{\mu}\right) h\left(X_{t \wedge \tau_{D}}\right)\right]=E_{x}\left[\exp \left(-\lambda^{+} A_{\tau_{D}}^{\mu}\right) h\left(X_{\tau_{D}}\right)\right]
$$

on account of the quasi-left continuity of $\mathbf{M}^{\alpha}$.

Lemma 4.13. The function $h$ satisfies

$$
h(x)=E_{x}\left[h\left(X_{\tau_{D}}\right)\right]-\lambda^{+} E_{x}\left[\int_{0}^{\tau_{D}} h\left(X_{s}\right) d A_{s}^{\mu}\right] .
$$

Proof. Since $h$ is $\mathcal{H}^{\lambda^{+} \mu}$-harmonic, for a bounded domain $D$,

$$
\lambda^{+} E_{x}\left[\int_{0}^{\tau_{D}} h\left(X_{t}\right) d A_{t}^{\mu}\right]=\lambda^{+} E_{x}\left[\int_{0}^{\tau_{D}} E_{X_{t}}\left(\exp \left(-\lambda^{+} A_{\tau_{D}}^{\mu}\right) h\left(X_{\tau_{D}}\right)\right) d A_{t}^{\mu}\right] .
$$

By the Markov property the right hand side equals

$$
\begin{aligned}
& \lambda^{+} E_{x}\left[\int_{0}^{\tau_{D}} \exp \left(\lambda^{+} A_{t}^{\mu}-\lambda^{+} A_{\tau_{D}}^{\mu}\right) h\left(X_{\tau_{D}}\right) d A_{t}^{\mu}\right] \\
& \quad=E_{x}\left[\exp \left(-\lambda^{+} A_{\tau_{D}}^{\mu}\right) h\left(X_{\tau_{D}}\right)\left(\exp \left(\lambda^{+} A_{\tau_{D}}^{\mu}\right)-1\right)\right] \\
& \quad=E_{x}\left[h\left(X_{\tau_{D}}\right)\right]-E_{x}\left[\exp \left(-\lambda^{+} A_{\tau_{D}}^{\mu}\right) h\left(X_{\tau_{D}}\right)\right],
\end{aligned}
$$

which implies (4.18).

By the same argument as in [4, Proposition 6.1], we see that the right hand side of (4.18) is continuous on $D$. Hence we have

Proposition 4.14. The function $h$ is continuous.

Now we consider asymptotic of $h$ as $|x| \rightarrow \infty$. Let $w$ be a positive continuous function with compact support. Suppose that $0 \in \operatorname{supp}[w] \subset B(R)$. By Theorem 4.8 and the continuity of $h$

$$
c \int_{B(R)} G^{\nu}(x, y) w(y) d y \leq h(x) \leq C \int_{B(R)} G^{\nu}(x, y) w(y) d y,
$$

and so by the inequality (4.5),

$$
c \int_{B(R)} G(x, y) w(y) d y \leq h(x) \leq C \int_{B(R)} G(x, y) w(y) d y .
$$

The Harnack inequality to $\{G(x, \cdot)\}_{\left\{x \in B(R)^{c}\right\}}$ says that for any $x \in B(R)^{c}$ and $y \in \operatorname{supp}[w]$

$$
c G(x, y) \leq G(x, 0) \leq C G(x, y) .
$$


Therefore we see that

$$
c G(x, 0) \leq h(x) \leq C G(x, 0) \text { for } x \in B(R)^{c},
$$

namely,

$$
\frac{c}{|x|^{d-\alpha}} \leq h(x) \leq \frac{C}{|x|^{d-\alpha}} \text { for } x \in B(R)^{c} .
$$

We call $\mathcal{H}^{\lambda^{+} \mu}$ positive (resp. null) critical if the ground state $h$ is in (resp. not in) $L^{2}(m)$. These notions are corresponding to the positive (resp. null) recurrence.

The equation (4.19) leads us to

Theorem 4.15. The operator $\mathcal{H}^{\lambda^{+} \mu}$ is null critical if and only if $\alpha<d \leq 2 \alpha$.

\section{An extension of Oshima's inequality}

In this section, we prove a functional inequality for critical Schrödinger forms. This inequality is regarded as a version of Oshima's inequality and plays a crucial role for the proof of the differentiability of $C(\lambda)$.

Lemma 5.1. Let $h$ be the $\mathcal{H}^{\lambda^{+} \mu}$-harmonic function constructed in the previous section. Then the h-transformed semigroup ${P_{t}^{\lambda^{+}} \mu, h}_{\text {of }}{P_{t}^{\lambda^{+}} \mu}^{\text {has }}$ the strong Feller property.

Proof. We follow the argument in [10, Corollary 5.2.7]. Let $f$ be a bounded Borel function and $\left\{x_{n}\right\}$ a sequence so that $x_{n} \rightarrow x$ as $n \rightarrow \infty$. Recall that $p^{\lambda^{+} \mu}(t, x, y)$ is jointly continuous ([2, Theorem 3.10]). Then by Fatou's lemma and the continuity of $h$,

$$
\begin{array}{r}
\liminf _{n \rightarrow \infty} \int_{\mathbb{R}^{d}} \frac{1}{h\left(x_{n}\right)} p^{\lambda^{+} \mu}\left(t, x_{n}, y\right) h(y)\left(\|f\|_{\infty} \pm f(y)\right) d y \\
\quad \geq \int_{\mathbb{R}^{d}} \frac{1}{h(x)} p^{\lambda^{+} \mu}(t, x, y) h(y)\left(\|f\|_{\infty} \pm f(y)\right) d y
\end{array}
$$

and thus the function,

$$
x \mapsto \int_{\mathbb{R}^{d}} \frac{1}{h(x)} p^{\lambda^{+} \mu}(t, x, y) h(y)\left(\|f\|_{\infty} \pm f(y)\right) d y,
$$

is lower semi-continuous. Note that $P_{t}^{\lambda^{+} \mu, h}$ is recurrent, in particular, conservative. Then

$$
\begin{aligned}
& \int_{\mathbb{R}^{d}} \frac{1}{h(x)} p^{\lambda^{+} \mu}(t, x, y) h(y) f(y) d y \\
= & \int_{\mathbb{R}^{d}} \frac{1}{h(x)} p^{\lambda^{+} \mu}(t, x, y) h(y)\left(\|f\|_{\infty}+f(y)\right) d y-\|f\|_{\infty} \\
= & -\int_{\mathbb{R}^{d}} \frac{1}{h(x)} p^{\lambda^{+} \mu}(t, x, y) h(y)\left(\|f\|_{\infty}-f(y)\right) d y+\|f\|_{\infty},
\end{aligned}
$$

and thus the function

$$
x \mapsto \int_{\mathbb{R}^{d}} \frac{1}{h(x)} p^{\lambda^{+} \mu}(t, x, y) h(y) f(y) d y,
$$

is lower and upper semi-continuous. 
Proposition 5.2. The h-transformed process $\mathbf{M}^{\lambda^{+} \mu, h}=\left(P_{x}^{\lambda^{+} \mu, h}, X_{t}\right)$ is Harris recurrent, that is, for a non-negative function $f$,

$$
\int_{0}^{\infty} f\left(X_{t}\right) d t=\infty \quad P_{x}^{\lambda^{+} \mu, h}-a . s .
$$

whenever $m(\{x: f(x)>0\})>0$.

Proof. Set $A=\{x: f(x)>0\}$. Since $P_{t}^{\lambda^{+} \mu, h}$ generates an $h^{2} m$-symmetric recurrent Markov process,

$$
P_{x}\left[\sigma_{A} \circ \theta_{n}<\infty, \forall n \geq 0\right]=1 \text { for q.e. } x \in \mathbb{R}^{d}
$$

by [12, Theorem 4.6]. Moreover, since the Markov process $\mathbf{M}^{\lambda^{+} \mu, h}$ has the transition density function

$$
\frac{p^{\lambda^{+} \mu}(t, x, y)}{h(x) h(y)}
$$

with respect to $h^{2} m,(5.2)$ holds for all $x \in \mathbb{R}^{d}$ by [12, Problem 4.6.3]. Using Lemma 5.1. (5.2), and the proof of [22, Chapter X, Proposition (3.11)], we see that $\mathbf{M}^{\lambda^{+} \mu, h}$ is Harris recurrent.

Theorem 5.3. There exist a positive function $g \in L^{1}\left(h^{2} m\right)$ and a function $\psi \in$ $C_{0}\left(\mathbb{R}^{d}\right)$ with $\int_{\mathbb{R}^{d}} \psi h^{2} d x=1$ such that

$$
\int_{\mathbb{R}^{d}}\left|u(x)-h(x) L\left(\frac{u}{h}\right)\right| g(x) h(x) d x \leq C \mathcal{E}^{\lambda^{+} \mu}(u, u)^{1 / 2}, u \in \mathcal{D}\left(\mathcal{E}^{\lambda^{+} \mu}\right),
$$

where

$$
L(u)=\int_{\mathbb{R}^{d}} u \psi h^{2} d x
$$

Proof. By Proposition 5.2, we can apply Oshima's inequality in [17] to the Dirichlet form $\left(\mathcal{E}^{\lambda^{+} \mu, h}, \mathcal{D}\left(\mathcal{E}^{\lambda^{+} \mu, h}\right)\right)$ satisfying the Harris recurrence condition; there exist a positive function $g \in L^{1}\left(h^{2} m\right)$ and a function $\psi \in C_{0}\left(\mathbb{R}^{d}\right)$ with $\int_{\mathbb{R}^{d}} \psi h^{2} d x=1$ such that

$$
\int_{\mathbb{R}^{d}}|u(x)-L(u)| g(x) h^{2}(x) d x \leq C \mathcal{E}^{\lambda^{+} \mu, h}(u, u)^{1 / 2}, \quad u \in \mathcal{D}\left(\mathcal{E}^{\lambda^{+} \mu, h}\right)
$$

where

$$
L(u)=\int_{\mathbb{R}^{d}} u \psi h^{2} d x
$$

Substituting $v / h$ for $u$ in (5.4) together with the equality

$$
\mathcal{E}^{\lambda^{+} \mu, h}(v, v)=\mathcal{E}^{\lambda^{+} \mu}(h v, h v),
$$

we obtain the equality (5.3). 


\section{Differentiability OF SPECTRAL FUnCTION}

Before proving the differentiability of spectral function, we prepare a lemma relevant to general regular Dirichlet forms.

Lemma 6.1. Let $X$ be a locally compact separable metric space, $m$ a positive Radon measure on $X$, and $(\mathcal{E}, \mathcal{D}(\mathcal{E}))$ a regular Dirichlet form on $L^{2}(X ; m)$. Let $\left\{u_{n}\right\} \subset \mathcal{D}(\mathcal{E})$ be a sequence with $\lim _{n \rightarrow \infty} \mathcal{E}\left(u_{n}, u_{n}\right)=0$ and $\lim _{n \rightarrow \infty} u_{n}=0$ m-a.e. Then there is a subsequence $\left\{u_{n_{k}}\right\}$ such that $\lim _{k \rightarrow \infty} u_{n_{k}}=0$ q.e.

Proof. Let $g$ be a non-negative continuous function with compact support and define

$$
\mathcal{E}^{g}(u, u)=\mathcal{E}(u, u)+\int_{X} u^{2} g d m .
$$

Then $\left(\mathcal{E}^{g}, \mathcal{D}\left(\mathcal{E}^{g}\right)(=\mathcal{D}(\mathcal{E}))\right)$ becomes a transient Dirichlet form. Let $u_{n}^{(l)}=((-l) \vee$ $\left.u_{n}\right) \wedge l, l=1,2, \cdots$. Then by the assumption $\lim _{n \rightarrow \infty} \mathcal{E}^{g}\left(u_{n}^{(l)}, u_{n}^{(l)}\right)=0$ for any $l$. Hence the 0 -order version of [12, Theorem 2.1.4] says that there exists a subsequence $\left\{u_{n_{k}}^{(l)}\right\}$ of $\left\{u_{n}^{(l)}\right\}$ such that $\lim _{k \rightarrow \infty} u_{n_{k}}^{(l)}=0 \mathrm{Cap}^{\mathrm{g},(0)}$-q.e. Here $\mathrm{Cap}^{\mathcal{E},(\mathrm{r})}$ denotes the $r$-order capacity with respect to $\left(\mathcal{E}^{g}, \mathcal{D}\left(\mathcal{E}^{g}\right)\right)$. Note that by [12, Theorem 2.1.6],

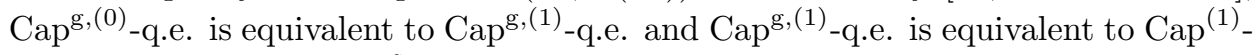
q.e. because $\mathcal{E}_{1}(u, u) \leq \mathcal{E}_{1}^{g}(u, u) \leq\left(1+\|g\|_{\infty}\right) \mathcal{E}_{1}(u, u)$.

Therefore we see that $\lim _{k \rightarrow \infty} u_{n_{k}}^{(l)}=0$ q.e. This proves the lemma because $l$ is arbitrary.

Theorem 6.2. Let $\mu=\mu^{+}-\mu^{-} \in \mathcal{K}_{d, \alpha}^{\infty}-\mathcal{K}_{d, \alpha}^{\infty}$. If $\alpha<d \leq 2 \alpha$, that is, $\mathcal{H}^{\lambda^{+} \mu}$ is null critical, then the spectral function $C(\lambda)$ is differentiable.

Proof. We deal with the case of $\lambda \geq 0$. First note that for $\lambda>\lambda^{+},-C(\lambda)$ is the principal eigenvalue of the operator $\mathcal{H}^{\lambda \mu}=\frac{1}{2}(-\Delta)^{\alpha / 2}-\lambda \mu([30$, Lemma 4.3]) and thus $C(\lambda)$ is differentiable by the analytic perturbation theory [13, Chapter VII]. Hence it is enough to prove the differentiability of $C(\lambda)$ at $\lambda=\lambda^{+}$. Furthermore, since $C(\lambda)$ is convex by the definition, we have only to prove the existence of a sequence $\left\{\lambda_{n}\right\}$ such that $d C\left(\lambda_{n}\right) / d \lambda \downarrow 0$ as $\lambda_{n} \downarrow \lambda^{+}$.

By [13, p.405, Chapter VII (4.44)], we see

$$
\frac{d C(\lambda)}{d \lambda}=-\int_{\mathbb{R}^{d}} u_{\lambda}^{2} d \mu>0, \quad \lambda>\lambda^{+}
$$

where $u_{\lambda}$ is the $L^{2}$-normalized eigenfunction corresponding to the eigenvalue $-C(\lambda)$, that is,

$$
-C(\lambda)=\mathcal{E}^{\lambda \mu}\left(u_{\lambda}, u_{\lambda}\right)=\lambda \int_{\mathbb{R}^{d}} u_{\lambda}^{2} d \mu+\mathcal{E}^{(\alpha)}\left(u_{\lambda}, u_{\lambda}\right) .
$$

Neglecting the positive part $\mu^{+}$of $\mu$ in the (6.3), we have

$$
\mathcal{E}^{(\alpha)}\left(u_{\lambda}, u_{\lambda}\right) \leq-C(\lambda)+\lambda \int_{\mathbb{R}^{d}} u_{\lambda}^{2} d \mu^{-}
$$

Furthermore, it follows from (3.5) that the right hand side above is dominated by

$$
-C(\lambda)+\lambda \epsilon \mathcal{E}^{(\alpha)}\left(u_{\lambda}, u_{\lambda}\right)+\lambda M(\epsilon) .
$$


Let $\left\{\lambda_{n}\right\}$ be a sequence with $\lim _{n \rightarrow \infty} \lambda_{n} \downarrow \lambda^{+}$. Substituting $\lambda_{n}$ for $\lambda$ in the equation above and taking $\epsilon>0$ so small that $\lambda_{n} \epsilon<1$, we have

$$
\mathcal{E}^{(\alpha)}\left(u_{\lambda_{n}}, u_{\lambda_{n}}\right) \leq \frac{-C\left(\lambda_{n}\right)+\lambda_{n} M(\epsilon)}{1-\lambda_{n} \epsilon},
$$

and thus

$$
\limsup _{n \rightarrow \infty} \mathcal{E}^{(\alpha)}\left(u_{\lambda_{n}}, u_{\lambda_{n}}\right)=\frac{\lambda^{+} M(\epsilon)}{1-\lambda^{+} \epsilon}<\infty
$$

because $C\left(\lambda_{n}\right) \rightarrow 0$ as $n \rightarrow \infty$. Since by (6.4)

$$
\begin{aligned}
& \left|\mathcal{E}^{\lambda^{+} \mu}\left(u_{\lambda_{n}}, u_{\lambda_{n}}\right)+C\left(\lambda_{n}\right)\right|=\left|\mathcal{E}^{\lambda^{+} \mu}\left(u_{\lambda_{n}}, u_{\lambda_{n}}\right)-\mathcal{E}^{\lambda_{n} \mu}\left(u_{\lambda_{n}}, u_{\lambda_{n}}\right)\right| \\
& \quad \leq\left(\lambda_{n}-\lambda^{+}\right) \int_{\mathbb{R}^{d}} u_{\lambda_{n}}^{2} d \mu \\
& \quad \leq\left(\lambda_{n}-\lambda^{+}\right)\left(\|G|\mu|\|_{\infty} \mathcal{E}^{(\alpha)}\left(u_{\lambda_{n}}, u_{\lambda_{n}}\right)\right) \longrightarrow 0
\end{aligned}
$$

as $n \rightarrow \infty$,

$$
\lim _{n \rightarrow \infty} \mathcal{E}^{\lambda^{+} \mu}\left(u_{\lambda_{n}}, u_{\lambda_{n}}\right)=0
$$

Let $h$ be the $\mathcal{H}^{\lambda^{+}} \mu$-harmonic function constructed in Section 4 and denote by $\left(\mathcal{E}^{\lambda^{+} \mu, h}, \mathcal{D}\left(\mathcal{E}^{\lambda^{+} \mu, h}\right)\right)$ the Dirichlet form of the $h$-transformed process. Then the equation (6.5) proves

$$
\lim _{n \rightarrow \infty} \mathcal{E}^{\lambda^{+} \mu, h}\left(\frac{u_{\lambda_{n}}}{h}, \frac{u_{\lambda_{n}}}{h}\right)=0 .
$$

Let $\psi$ and $L(u)$ be the things in Theorem 5.3 . Then since

$$
\begin{aligned}
\left|L\left(\frac{u_{\lambda_{n}}}{h}\right)\right| & =\int_{\mathbb{R}^{d}} u_{\lambda_{n}}(x) \psi(x) h(x) d x \\
& \leq \sqrt{\int_{\mathbb{R}^{d}} u_{\lambda_{n}}^{2} d x} \sqrt{\int_{\mathbb{R}^{d}} \psi^{2}(x) h^{2}(x) d x}<\infty,
\end{aligned}
$$

we may assume that $L\left(u_{\lambda_{n}} / h\right)$ converges to a certain constant $C$ by taking a subsequence of $\left\{\lambda_{n}\right\}$ if necessary. In addition, since (5.3) says

$$
\begin{aligned}
\int_{\mathbb{R}^{d}}\left|u_{\lambda_{n}}-C h\right| g h d x & \leq \int_{\mathbb{R}^{d}}\left|u_{\lambda_{n}}-h L\left(\frac{u_{\lambda_{n}}}{h}\right)\right| g h d x+\int_{\mathbb{R}^{d}}\left|h L\left(\frac{u_{\lambda_{n}}}{h}\right)-C h\right| g h d x \\
& \leq C \mathcal{E}^{\lambda^{+} \mu}\left(u_{\lambda_{n}}, u_{\lambda_{n}}\right)^{1 / 2}+\int_{\mathbb{R}^{d}}\left|L\left(\frac{u_{\lambda_{n}}}{h}\right)-C\right| g h^{2} d x \rightarrow 0,
\end{aligned}
$$

we may assume that $u_{\lambda_{n}} \rightarrow C h m$-a.e. Now recall that $\mathcal{H}^{\lambda^{+}} \mu$ is null critical if and only if $d \leq 2 \alpha$. Then the constant $C$ must be equal to 0 because

$$
1=\liminf _{n \rightarrow \infty} \int_{\mathbb{R}^{d}} u_{\lambda_{n}}^{2} d x \geq \int_{\mathbb{R}^{d}} \liminf _{n \rightarrow \infty} u_{\lambda_{n}}^{2} d x=C^{2} \int_{\mathbb{R}^{d}} h^{2} d x
$$

and consequently

$$
\lim _{n \rightarrow \infty} u_{\lambda_{n}}=0, \quad m \text {-a.e. }
$$

Notice that $\mathcal{E}^{\lambda^{+} \mu, h}$-q.e. is equivalent to $\mathcal{E}^{(\alpha)}$-q.e. Then combing (6.6) and (6.8) with Lemma 6.1, we may assume that $u_{\lambda_{n}}$ converges to 0 q.e.

Since $u_{\lambda_{n}}$ is the eigenfunction corresponding to $C\left(\lambda_{n}\right)$,

$$
u_{\lambda_{n}}=e^{-C\left(\lambda_{n}\right) t} P_{t}^{\lambda_{n} \mu} u_{\lambda_{n}}
$$


and

$$
\left\|u_{\lambda_{n}}\right\|_{\infty} \leq e^{-C\left(\lambda_{n}\right) t}\left\|P_{t}^{-\lambda_{n} \mu^{-}}\right\|_{2, \infty} \leq\left\|P_{t}^{-\lambda_{1} \mu^{-}}\right\|_{2, \infty}<\infty
$$

by [2, Theorem 6.1 (iii)]. Hence we have

$$
\begin{aligned}
\limsup _{n \rightarrow \infty}\left|\int_{\mathbb{R}^{d}} u_{\lambda_{n}}^{2} d \mu\right| & \leq \limsup _{n \rightarrow \infty} \int_{\mathbb{R}^{d}} u_{\lambda_{n}}^{2} d|\mu| \\
& =\limsup _{n \rightarrow \infty}\left(\int_{\mathbb{R}^{d}} u_{\lambda_{n}}^{2} d|\mu|_{R}+\int_{\mathbb{R}^{d}} u_{\lambda_{n}}^{2} d|\mu|_{R^{c}}\right) \\
& \leq \limsup _{n \rightarrow \infty} \int_{\mathbb{R}^{d}} u_{\lambda_{n}}^{2} d|\mu|_{R}+\limsup _{n \rightarrow \infty}\left\|G|\mu|_{R^{c}}\right\|_{\infty} \mathcal{E}^{(\alpha)}\left(u_{\lambda_{n}}, u_{\lambda_{n}}\right) \\
& \leq\left\|G|\mu|_{R^{c}}\right\|_{\infty} \frac{\lambda^{+} M(\epsilon)}{1-\lambda^{+} \epsilon} .
\end{aligned}
$$

By letting $R$ to $\infty$, we complete the proof.

Remark 6.3. When $\alpha=2$ and the potential $\mu$ is absolutely continuous with respect to the Lebesgue measure, non-differentiability of the spectral function was considered in [24]. The argument in [24, Theorem 2.1] can be adapted to prove that if $d>2 \alpha$, then $C(\lambda)$ is not differentiable. Indeed, the ground state $h$ belongs to $L^{2}(m)$, that is, zero is an eigenvalue of $\mathcal{H}^{\lambda^{+} \mu}$. We normalize the function $h$ as $\|h\|_{2}=1$. Let $\left\{u_{\lambda_{n}}\right\}$ be the sequence defined in the proof of Theorem 6.2. that is, $u_{\lambda_{n}}$ is the $L^{2}(m)$-normalized eigenfunction corresponding with the eigenvalue $\lambda_{n}$ $\left(\lambda_{n}>\lambda^{+}\right)$. Since $\left\{u_{\lambda_{n}}\right\}$ is bounded in $\mathcal{E}^{(\alpha)}$ and in $L^{2}(m)$, we may suppose that

$$
u_{\lambda_{n}} \rightarrow u_{0}, \quad \text { weakly in } \mathcal{E}^{(\alpha)} \text { and in } L^{2}(m) .
$$

Moreover we know in the proof of Theorem 6.2 that

$$
u_{\lambda_{n}} \rightarrow C h, m \text {-a.e. }
$$

Hence $u_{0}=C h, m$-a.e., and thus the constant $C$ is less than or equal to 1 . Since for $\lambda>\lambda^{+}$,

$$
-C(\lambda) \leq \mathcal{E}^{(\alpha)}(h, h)+\lambda \int h^{2} d \mu
$$

and

we have

$$
\mathcal{E}^{(\alpha)}(h, h)=-\lambda^{+} \int_{\mathbb{R}^{d}} h^{2} d \mu
$$

$$
\frac{C(\lambda)}{\lambda-\lambda^{+}} \geq-\int h^{2} d \mu
$$

Noting that there exists a constant $\theta \in(0,1)$ such that

$$
\frac{C(\lambda)}{\lambda-\lambda^{+}}=C^{\prime}\left(\lambda^{+}+\theta\left(\lambda-\lambda^{+}\right)\right)
$$

by the mean value theorem, we can find a sequence $\left\{a_{n}\right\}$ such that $a_{n} \rightarrow \lambda^{+}$as $n \rightarrow \infty$ and

By Theorem 3.4,

$$
\limsup _{\lambda \rightarrow \lambda^{+}} \frac{C(\lambda)}{\lambda-\lambda^{+}}=\lim _{n \rightarrow \infty} C^{\prime}\left(a_{n}\right) \text {. }
$$

$$
\lim _{n \rightarrow \infty} C^{\prime}\left(a_{n}\right)=-\lim _{n \rightarrow \infty} \int u_{a_{n}}^{2} d \mu=-C^{2} \int_{\mathbb{R}^{d}} h^{2} d \mu .
$$


Since $h>0 \in \mathcal{D}_{e}\left(\mathcal{E}^{(\alpha)}\right)$ and $\left(\mathcal{E}^{(\alpha)}, \mathcal{D}_{e}\left(\mathcal{E}^{(\alpha)}\right)\right)$ is a Hilbert space, we know that

$$
-\int_{\mathbb{R}^{d}} h^{2} d \mu=\frac{1}{\lambda^{+}} \mathcal{E}^{(\alpha)}(h, h)>0 .
$$

Hence

$$
\limsup _{\lambda \rightarrow \lambda^{+}} \frac{C(\lambda)}{\lambda-\lambda^{+}} \leq-C^{2} \int_{\mathbb{R}^{d}} h^{2} d \mu \leq-\int_{\mathbb{R}^{d}} h^{2} d \mu .
$$

On the other hand, by (6.9), we find that

$$
\liminf _{\lambda \rightarrow \lambda^{+}} \frac{C(\lambda)}{\lambda-\lambda^{+}} \geq-\int_{\mathbb{R}^{d}} h^{2} d \mu .
$$

Therefore

$$
\lim _{\lambda \rightarrow \lambda^{+}} \frac{C(\lambda)}{\lambda-\lambda^{+}}=-\int_{\mathbb{R}^{d}} h^{2} d \mu>0 .
$$

\section{ACKNOWLEDGMENTS}

We thank Z.-Q. Chen for valuable comments and remarks that helped to improve the manuscript. We also thank an anonymous referee for a careful reading of this paper and many helpful comments.

\section{REFERENCES}

1. Aizenman, M., Simon, B.: Brownian motion and Harnack inequality for Schrödinger operators, Comm. Pure Appl. Math. 35, 209-273, (1982). MR0644024 (84a:35062)

2. Albeverio, S., Blanchard, P., Ma, Z.M.: Feynman-Kac semigroups in terms of signed smooth measures, in "Random Partial Differential Equations" ed. U. Hornung et al., Birkhäuser, (1991). MR.1185735 (93i:60140)

3. Arendt, W., Batty, C.J.K.: The spectral function and principal eigenvalues for Schrödinger operators, Potential Anal. 7, 415-436, (1997). MR1462579 (98g:35148)

4. Bogdan, K., Byczkowski, T.: Potential theory of Schrödinger operator based on fractional Laplacian, Probability and Mathematical Statistics 20, 293-335, (2000). MR.1825645 (2002a:31002)

5. Bass, R., Levin, D.A. : Harnack inequalities for jump processes, Potential Anal. 17, 375-388, (2002). MR 1918242 (2003e:60194)

6. Boukricha, A., Hansen, W., Hueber, H.: Continuous solutions of the generalized Schrödinger equation and perturbation of harmonic spaces, Expo. Math. 5, 97-135, (1987). MR0887788 (88g:31019)

7. Chen, Z.-Q.: Gaugeability and Conditional Gaugeability, Trans. Ameri. Math. Soc. 354, 4639-4679, (2002). MR.1926893 (2003i:60127)

8. Chen, Z.-Q., Song, R.: Estimates on Green functions and Poisson kernels for symmetric stable processes, Math. Ann. 312, 465-501, (1998). MR.1654824 (2000b:60179)

9. Davies, E.B., One Parameter Semigroup, London Mathematical Society Monographs, Academic Press, (1980). MR0591851 (82i:47060)

10. Davies, E.B., Heat Kernels and Spectral Theory, Cambridge Univ. Press, Cambridge, U.K., (1989). MR.0990239 (90e:35123)

11. Dembo, A., Zeitouni, O., Large deviation techniques and applications, Second edition, Applications of Mathematics 38, Springer-Verlag, New York, (1998). MR1619036 (99d:60030)

12. Fukushima, M., Oshima, Y., Takeda, M.: Dirichlet Forms and Symmetric Markov Processes, Walter de Gruyter, Berlin, (1994). MR 1303354(96f:60126)

13. Kato, T.: Perturbation Theory for Linear Operators, Springer-Verlag, Berlin-Heidelberg-New York, (1984). MR1335452 (96a:47025)

14. Lieb E.H. and Loss M.: Analysis, Second edition, Graduate Studies in Math. 14, American Mathematical Society, (2001). MR1817225 (2001i:00001)

15. Maz'ja, V.G.: Sobolev Spaces, Springer, (1985). MR0817985 (87g:46056)

16. Murata, M.: Structure of positive solutions to $(-\Delta+V) u=0$ in $R^{n}$, Duke Math. J. 53, 869-943, (1986). MR0874676 (88f:35039) 
17. Oshima, Y.: Potential of recurrent symmetric Markov processes and its associated Dirichlet spaces, in Functional Analysis in Markov Processes, ed. M. Fukushima, Lecture Notes in Math. 923, Springer-Verlag, Berlin-Heidelberg-New York, (1982). MR0661629 (85k:60107)

18. Pinchover, Y.: Criticality and ground states for second-order elliptic equations, J. Differential Equations 80, 237-250, (1989). MR1011149 (91c:35046)

19. Pinsky, R.G.: Positive Harmonic Functions and Diffusion, Cambridge Studies in Advanced Mathematics 45, Cambridge University Press, (1995). MR.1326606 (96m:60179)

20. Reed, M., Simon, B.: Methods of Modern Mathematical Physics, Volume I, Functional Analysis, Academic Press, (1972). MR0493419(58:12429a)

21. Reed, M., Simon, B.: Methods of Modern Mathematical Physics, Volume IV, Analysis of Operators, Academic Press, (1979).

22. Revuz, D., Yor, M.: Continuous Martingales and Brownian Motion, 3rd edition, Springer, New York, (1998). MR.1725357 (2000h:60050)

23. Silverstein, M. L.: Symmetric Markov processes, Lecture Notes in Mathematics 426, SpringerVerlag, Berlin-New York, (1974). MR0386032 (52:6891)

24. Simon, B.: On the absorption of eigenvalues by continuous spectrum in regular perturbation problems, J. Funct. Anal. 25, 338-344, (1977). MR0445317 (56:3659)

25. Stollmann, P., Voigt, J.: Perturbation of Dirichlet forms by measures, Potential Anal. 5, 109-138, (1996). MR1378151 (97e:47065)

26. Takeda, M.: Asymptotic properties of generalized Feynman-Kac functionals, Potential Analysis 9, 261-291, (1998). MR.1666887(2000a:60049)

27. Takeda, M.: Exponential decay of lifetimes and a theorem of Kac on total occupation times, Potential Analysis 11, 235-247, (1999). MR.1717103 (2000i:60084)

28. Takeda, M.: $L^{p}$-independence of the spectral radius of symmetric Markov semigroups, Stochastic Processes, Physics and Geometry: New Interplays. II: A Volume in Honor of Sergio Albeverio, Edited by Fritz Gesztesy, et al., (2000). MR1803452 (2002a:47070)

29. Takeda, M.: Conditional gaugeability and subcriticality of generalized Schrödinger operators, J. Funct. Anal. 191, 343-376, (2002). MR.1911190(2003e:60176)

30. Takeda, M.: Large deviation principle for additive functionals of Brownian motion corresponding to Kato measures, Potential Analysis, 19, 51-67, (2003). MR1962951(2003k:60060)

31. Takeda, M., Tsuchida, K.: Criticality of generalized Schrödinger operators and differentiability of spectral functions, Advanced Studies in Pure Mathematics 41. Math. Soc. of Japan, Tokyo, 333-350, (2004). MR2083718 (2005h:60240)

32. Takeda, M., Uemura, T.: Subcriticality and Gaugeability for Symmetric $\alpha$-Stable Processes, Forum Math. 16, 505-517, (2004). MR2044025 (2005d:60124)

33. Wu,L.: Exponential convergence in probability for empirical means of Brownian motion and random walks, J. Theoretical Probab. 12, 661-673, (1999). MR.1702907 (2001g:60062)

34. Zhao, Z.: A probabilistic principle and generalized Schrödinger perturbation. J. Funct. Anal. 101, 162-176, (1991). MR1132313 (93f:60116)

35. Zhao, Z.: Subcriticality and gaugeability of the Schrödinger operator, Trans. Amer. Math. Soc. 334, 75-96, (1992). MR1068934(93a:81041)

Mathematical Institute, Tohoku University, Aoba, Sendai, 980-8578, Japan

E-mail address: takeda@math.tohoku.ac.jp

Mathematical Institute, Tohoku University, Aoba, Sendai, 980-8578, Japan

E-mail address: kanedon@ma8.seikyou.ne.jp 